\title{
7 Appendix II: Synopses of textual variants
}

\subsection{Ibn Zurayq, al-Qașīda al-Andalusiyya}

The copy of reference is the printed version included in -Safadī, Wāfì 21,112-15. Variants are arranged in the order of their frequency. The manuscripts referred to all belong to the Staatsbibliothek zu Berlin-Preussischer Kulturbesitz. It may be noted that there is a high degree of text stability throughout the manuscripts of Ibn Zurayq's poem.

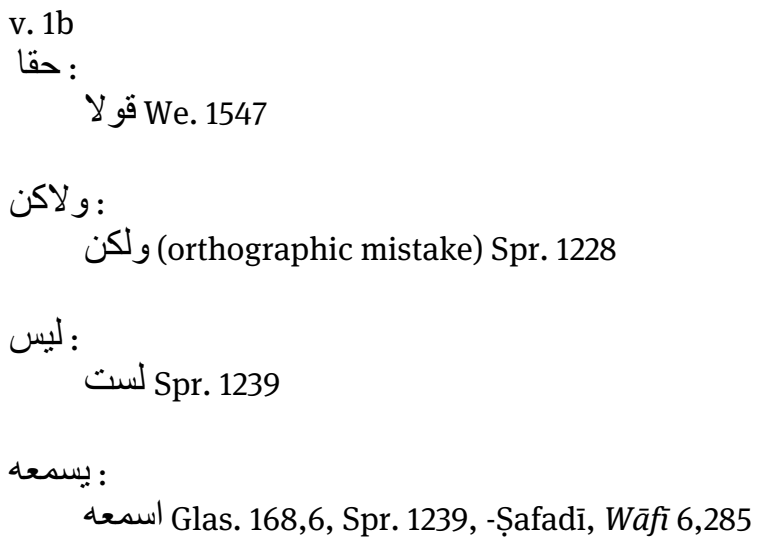

V. $2 \mathrm{~b}$

: اللوم :

Mq. 117, Lb. 243

v. $3 a$

فq. 674, Lb. 243a, Pm. 193,2, Glas. 37, Spr. 1239

Spr. 1228, Mq. 117 
Spr. 1228

: تانبيه

Spr. 1228

Spr. 1239

v. $3 \mathrm{~b}$

: من عسفه :

Spr. 1228

We. 1547

من عن عن عن عذله عنه

Spr. 1239

v. $4 \mathrm{a}$

: بالخطب

We. 1547, Pm. 193,1, 193,2, Glas. 37, 168,6, Spr. 1239

Spr. 1228

v. $4 \mathrm{~b}$

: بخطوب البين

Spr. 1228

من We. 1547

من من خطوب البين Pm. 193,2

: البين

Mq. 117

v. $5 a$

: بكفيك :

We. 1547, Spr. 1228, 1239, Mq. 117, 674, Lb. 243a, Pm. 193,1, 193,2, 542

Glas. 37, 168

روعة:

We. 1547, Mq. 117, 674, Lb. 243a, Pm. 193,2, 542, Glas. 168,6, Spr. 1239

:

Spr. 1228

Mq. 117 
Mq. 674

V. $6 \mathrm{~b}$

Glas. 37 (variant in the margin):

بالعزم Pm. 193,1, Glas. 37, 168(?)

: يجمعه

Spr. 1228, Pm. 193,2

v. $7 \mathrm{a}$

: تابى :

يابى Mq. 674, fo. 167r, 1.3

Mq. 674, fo. 167r , 1.6

تابا Glas. 37

Spr. 1239

Lb. 243a, Pm. 193,1, 193,2, 542, Glas. 37, 168, Spr. 1239:

S.Safadī, Wãfi, We. 1547

: تجشمه : تجمه

Spr. 1239

v. $7 \mathrm{~b}$

:

Pm. 542

كدحا :

لدا كدا 117

كدا Lb. 243a, Pm. 193,1, Glas. 37, 168

Spr. 1239

من:

Spr. 1239 كمن(؟)

: بودعه :

تودعه Mq. 117

We. 1547

Spr. 1239 
Pm. 193,2 includes an apocryphal hemistich in v. 7b:

اثشياء تخفضه طورا وترفعه

v. $8 \mathrm{a}$

كانما:

كانّ ما (irregular orthography) Pm. 542

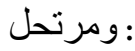

(orthographic mistake?) Pm. 193,1

v. $8 \mathrm{~b}$

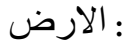

Spr. 1228

: بذر عه : بذه

Mq. 674

يجمعه (lapsus oculi? cf. v. 6b) Glas. 168

v. 9a

: اذا ما الزماع

اذ الزماع Pm. 193,1, Glas. 37

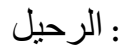

Pm. 542

: في الرحيل

Spr. 1239

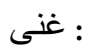

غنا Glas. 168, Spr. 1239

v. $9 \mathrm{~b}$

: السند :

Mq. 117, Lb. 243a, Pm. 193,2, Spr. 1239

الهند Pm. 193,1

مربع : مع d

Mq. 117, Glas. 168

يز Lb. 243a 
v. $10 a$

L :

فما We. 1547, Pm. 193,2

: واصلة

Mq. 674, Pm. 542

Glas. 168

Spr. 1239 موصولة

v. 11a

:وزع :

We. 1547, Mq. 117, 674, Lb. 243a, Pm. 542

: قد وز ع الله

الله

الله قسم

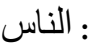

We. 1547, Mq. 117, 674, Lb. 243a, Glas. 37, 168, Spr. 1239

v. $11 \mathrm{~b}$

: بخلق :

Spr. 1228

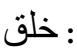

We. 1547, Spr. 1228, 1239, Mq. 674, Pm. 542, Glas. 37, 168

v. $12 \mathrm{a}$

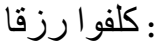

ملئو ا حرصا :Mq. 117, 674, (ملئو الخ) Lb. 243a, Pm. 193,1, 193,2, 542, Glas. 37, 168, Spr. 1239

: ملست :

Spr. 1239

:

I Pm. 193,2 
v. $12 \mathrm{~b}$

نقع : تعنه

يقنعه Spr. 1228, Mq. 117

(؟نفعه Pm. 542, Spr. 1239

v. $13 a$

فالحرص Pm. 193,2:

We. 1547 و الحرص

Pm. 193,1

Mq. 674

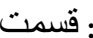

ضنمنت Spr. 1228

v. $13 \mathrm{~b}$

لا

We. 1547, Glas. 168,6

بغي

Spr. 1228

Spr. 1239

v. $14 \mathrm{a}$

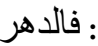

فاله ف فاله

بنه : منيث يمنعه :

Mq. 674, Pm. 193,1, 542, Glas. 37, 168,6, Spr. 1239

V. $14 \mathrm{~b}$

اربا

ارثا We. 1547

Mو. 674, Pm. 193,1, Glas. 37, 168,6, Spr. 1239

يو Pm. 542

منه (منة Pm. 193,2

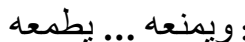

(inversion of word order) Pm. 193,1, 193,2, Glas. 37

Glas. 168,6, Spr. 1239 


\section{v. $15 \mathrm{a}$}

: استود ع الله

Mq. 117

(بغداد

بعددا بغذاذ (misspelling) Spr. 1228

بغذاذ (misspelling) Spr. 1239

v. 16a

ان :

لو Mq. 117, 674, Lb. 243a, Pm. 193,1, 542, Spr. 1239

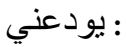

We. 1547, Spr. 1239

Mq. 117, Pm. 193,1

v. $16 \mathrm{~b}$

: صفو :

حو We. 1547

Spr. 1228, Lb. 243a, Spr. 1239

ريب Mq. 117

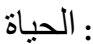

(المنون(؟ Mq. 117

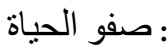

Pm. 193,1

v. $17 \mathrm{a}$

كم قد Lb. 243a, Pm. 193,1, 193,2:

Mq. 117

:

We. 1547, Mq. 674, Pm. 193,1, 542, Spr. 1239

Spr. 1228

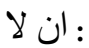

الان Mq. 117 
Glas. 168,6

v. $17 \mathrm{~b}$

: للضرورة

للضرور ات Lb. 243a, Pm. 193,1, 193 Glas. 37,2, Spr. 1239

للضرور اة (orthographic mistake) Pm. 542

حال :

حالا Lb. 243a

:تشفعه : تشع

ثُفعمه Spr. 1228

V. $18 \mathrm{a}$

: و كم تشبرث

Pm. 193,2

: في

• We. 1547, Mq. 674, Lb. 243a, Pm. 193,1, 542, Spr. 1239

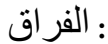

Mq. 117, 674, We. 1547, Spr. 1228, Lb. 243a

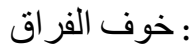

We. 1547, Spr. 1228, 1239, Mq. 674, Lb. 243a, Pm. 193,1, 193,2 (correction

in the margin), 542, Glas. 37, 168,6

v. $19 \mathrm{a}$

:

Spr. 1228

We. 1547

: منخرق

Spr. 1228 منخر قا

V. $19 \mathrm{~b}$

: بقر قته

We. 1547, Spr. 1228 
: كن

We. 1547 ولكن

v. $20 a$

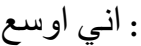

We. 1547 لاني لاوسع

اني لاوسع Pm. 193,2

: جنابته

Mq. 674, Pm. 542

v. $20 \mathrm{~b}$

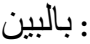

Spr. 1228

( ) : عني :

عنه Mq. 674, Pm. 193,1, 542

: جرمي

فو 1547

قُلبي

Glas. 37

: بوسعه : بوسه

(variant written above the line) Glas. 37

v. $21 \mathrm{a}$

رزت :

Mq. 674, Pm. 193,1, 193,2, 542, Glas. 37, 168,6, Spr. 1239

: فلم :

Mq. 117, Pm. 193,1, 193,2

v. $21 \mathrm{~b}$

: وكن من

We. 1547

كذالك من Mq. 117, Lb. 243a, Pm. 193,1 
v. $22 \mathrm{~b}$

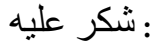

Lb. 243a

Mq. 674, Pm. 542

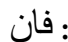

فعنه Mq. 117, 674, Pm. 193,1, 542, Glas. 37, 168

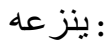

Spr. 1228, Pm. 193,1

v. $23 a$

اعتضت : اعتصنت

اعتصت اعنص Lb. 243a

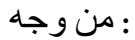

We 1547, Mq. 674, Pm. 193,2, 542

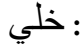

خل (defective orthography) Pm. 193,1

Mq. 117, Lb. 243a

حنب Pm. 193,2

: بعد :

عند Mq. 117, Lb. 243a

v. $23 \mathrm{~b}$

: كاسا :

كاس Lb. 243a

(تجرع

اجرع We 1547, Mq. 117, 674, Lb. 243a, Pm. 542

Pm. 193,2

: اجر : عه :

Mq. 117, Lb. 243a, Glas. 37

اتجر عه Pm. 193,2 
v. $24 \mathrm{a}$

ق قائل

قال We 1547

: لي :

لك Mq. 117, Lb. 243a

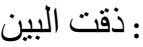

We 1547

Mq. 117, 674, Lb. 243a, Pm. 193,2, 542

: قلت :

قد صدقو 117

v. $24 \mathrm{~b}$

ادجعه : ادفعه :

الهجعه ادوعه Pm. 193,1

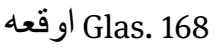

v. $25 \mathrm{a}$

VI:

هل Mq. 674, Pm. 542

اني Pm. 193,2

و وكان Mq. 117, 674, Pm. 542:

فكان Pm. 193,1

مكان Lb. 243a, Spr. 1239

v. $25 \mathrm{~b}$

-

حين Mq. 117, Lb. 243a

v. $26 \mathrm{a}$

: اني لاقطع

(metrically impossible) Lb. 243a

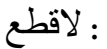

لا اقطع 1547 
فوها : انفدها

انفذها Pm. 193,2, 542, Glas. 168

v. $26 \mathrm{~b}$

: في حسرة

Glas. 37

v. $27 \mathrm{a}$

: با من :

Spr. 1228

ب.

Glas. 37

4:

Spr. 1228

v. $27 \mathrm{~b}$

: بلوعة

Spr. 1228 بلومة (؟) بله

حزنا عليه Lb. 243a

v. $28 \mathrm{a}$

: يطمنّن

We 1547

: لجنبي

لقلبي Pm. 542, Glas. 37

: مضجع :

Spr. 1228 مطجع

: كذا

Glas. 168,6

v. $28 \mathrm{~b}$

: يطمئن

We 1547

Glas. 37 
4.

بb. 243a

بنت

غبت Spr. 1228, Lb. 243a

Spr. 1239

: مضجعه :

مجمع Pm. 542

v. $29 \mathrm{a}$

ريب:

ان Spr. 1228, Mq. 117, 674, Lb. 243a, Pm. 542

v. $29 \mathrm{~b}$

إن

ظنّ Spr. 1228, Mq. 117

اظن Lb. 243a

v. $30 \mathrm{a}$

: جرى :

رمى :Pm. 193,2

ه - البين

Mq. 117, 674, Pm. 542, Spr. 1239

:بيدٍ :

Spr. 1228

v. $30 \mathrm{~b}$

عسر : عسراء :

عسر (orthographic mistake) Spr. 1239

عبر اء (misspelling?) Pm. 193,2

: حظي :

Glas. 168 حصلي (؟) 
v. $31 \mathrm{a}$

فكنت :

We. 1547, Lb. 243a, Pm. 193,1, 193,2

جاز عا : بداز

ابدا We. 1547, Pm. 193,2

ابديعا؟Lb. 243a

: مرقا

قلقا Mq. 117, Lb. 243a

v. $31 \mathrm{~b}$

: اجز عه :

We. 1547

v. $32 \mathrm{a}$

: القصف :

We. 1547, Glas. 37, 168, Spr. 1239

الوصل Pm. 193,2

درست : درسب :

دست

v. $32 \mathrm{~b}$

: اثثاره :

اياته Mq. 117

ايامه Lb. 243a

بنت

غبت Pm. 542

V. $33 \mathrm{~b}$

: التي

الذي We. 1547, Spr. 1239

: امضته :

Glas. 37, 168

Spr. 1239 
: ترجعه :

Spr. 1228

v. $34 \mathrm{~b}$

غيثن :

غيث We. 1547, Mq. 674, Lb. 243a, Pm. 193,1, 193,2, 542, Glas. 37, 168, Spr. 1239

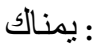

مغنالك We. 1547, Mq. 117, 674, Pm. 193,2, 542, Glas. 37, 168, Spr. 1239

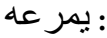

Pm. 193,2

v. $35 \mathrm{a}$

عهد : ع :

Glas. 168

عهدا لم Pm. 193,2

: بضبعه :

Spr. 1228

v. $35 \mathrm{~b}$

كما له :

كما عندي Mq. 117

كما عندي لن ألن Lb. 243a, Pm. 193,1

اضنبعه :

Spr. 1228, Pm. 542

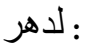

Mq. 117

v. $37 \mathrm{~b}$

: بـه و لا بي في حال بمتّعَه

Pm. 193,2, Glas. 168, Spr. 1239; the same var. also in a manuscript of -Subkī's Ṭabaqāt referred to in -Ṣafadì, p. 115, fn. 1 


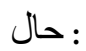

Spr. 1228

v. 38a

علم:

عq. 117, Lb. 243a?

S معقب : معب :

Sعقبا Spr. 1228, Pm. 193,2

فرجا Spr. 1239:

فرحا Mq. 117, 674, Lb. 243a, Pm. 193,1

v. 38b

: فاضيق :

Mq. 674, Pm. 542

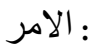

We. 1547

v. 39a

( على :

عليّ Mq. 674, Pm. 542

:

الذي (grammatically impossible?) Spr. 1239

(اضنت

اطنت We. 1547

v. 39b

( )

We. 1547, Spr. 1228, 1239, Lb. 243a, Pm. 193,1, 193,2, 542

Glas. 37, 168

v. $40 \mathrm{a}$

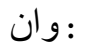

فُ Mq. 117 
: تغل : ت

We. 1547, Mq. 117, 674, Lb. 243a, Pm. 193,1, 542, Glas. 37, 168

احدا :

و و احدا We. 1547

v. $40 \mathrm{~b}$

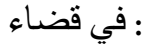

بقضنًاء Mq. 117, 674, Lb. 243a, Pm. 542

لقضاء الله Pm. 193,2

: يصنعه

نصن Mq. 117

Pm. 193,2

\section{2 -Ūshī, Qaṣīdat Bad' al-amālī}

Ms. Ahlwardt no. 2408/1 (= SBB 2408/1), containing the highest number of verses, although not constituting the "democratic version", ${ }^{185}$ has been used as reference text with which the other manuscripts have been compared.

The markings employed aim at illustrating the distinction between those variants which result from written transmission and those which may be explained through oral transmission. Bold markings indicate real variants; secondary variants are left lean, scribal errors (misspellings) are underlined.

V. 3a

مليك SBB 2408/1:

اله (ilāhun) (semantically and metrically possible) SBB 2408/3, 2408/6; Pri 2260/13; BSB 1735 (الان)

V. $4 \mathrm{a}$

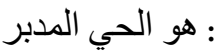

SBB 2408/6 هي المدبر هو هو

SBB 4944

185 The manuscript which has a sequence of verses shared by a majority of copies. 
V. 4b

: ذلو الجلان

ذي الجلالي (gramm. imposs.) Gött.

(sem. impos.) SBB 4944

V. $5 \mathrm{a}$

: القبيح :

قبيح (gramm. imposs.) SBB 4944

V. $6 a$

ليست : لينت

ليس (gramm. imposs.) SBB 4944

V. 6b

غير :

ذعينا BSB 1735

ذا الغضال (misspelling):

ذا ذانفصال Gött., SBB 2407, 2408/2, 2408/3, 2408/4, 2408/5, 2408/6, 4496, 4505, 4950; Pri 2260/2, 2260/7, 2260/8, 2260/11, 2260/13, 2264/4, 2272/4, 2272/8; BSB 1735

SBB 4944

V. 7a

: مفات الذات...

صفات الز اة ...طر (misspelling) SBB 4944

V. 7b

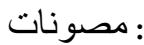

SBB 2408/5 (metrically impossible)

V. 8a

: نسمي .

BSB 1735

SBB 2408/1, 2407; Pri 2272/4, 2273:

(metrically imposs.) Gött., SBB 2408/3, 2408/4, 4496; Pri 2260/7, 2260/11,

2260/13, 2264/4, 2272/8 
كثيئً (gramm. imposs.) SBB 2408/6, 4505

V. $8 \mathrm{~b}$

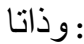

SBB 2408/4

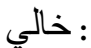

خالٍ (defective writing) Gött.

حال SBB 4944; Pri 2272/8

V. 9a

: غير اللمسمي :

(gramm. imposs.) SBB 2408/6 غير المسمي : للمسمي

V. $9 \mathrm{~b}$

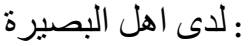

Pri 2273

Pri 2264/4 للدى اهل البصرة البهيرة

V. 10a

و ما ان (a pleonastic expression):

و واء (irregular orthography; perhaps a hearing mistake - the scribe may not have been

familiar with the expression) SBB 2407

وماعِن (irregular orthography) Pri 2260/13

V. $10 \mathrm{~b}$

: ذالإل

ذو الاشتمال (metrically imposs.) SBB 4950

BSB 1735 ذو الاشتمال

V. $12 \mathrm{a}$

مخلوقاً (grammatically wrong):

Gött.

V. $12 \mathrm{~b}$

: عن جنس المقال

SBB 2408/6 غير جنس المقال جن 
V. $14 \mathrm{~b}$

فُفن (!):

فصن Gött., SBB 2407, 2408/2, 2408/4, 2408/5, 2408/6, 4505, 4944; 4950; Pri 2260/2, 2260/7, 2260/8, 2260/11, 2260/13, 2264/4, 2272/4, 2272/8, 2273; BSB 1735

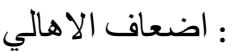

Gött., SBB 2407, 2408/2, 2408/3, 2408/5, 2408/6 (ني الاناف الاهالي :...), 4505; Pri 2260/2, 2260/7, 2260/11, 2260/13, 2264/4, 2272/4, 2272/8, 2273 SBB 2408/4

V. $15 a$

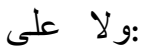

BSB 1735

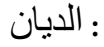

(misspelling) Pri 2260/7

V. $15 b$

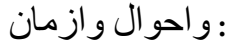

(word order inverted; metrically and semantically possible) SBB 2408/4

V. $17 \mathrm{a}$

: نصر :

نصير (Gött., metrically impossible),

SBB 4944

V. 17b

: ذو و الجلالة و المعالي

SBB 2407, 2408/2, 2408/3, 2408/5, 2408/6, 4496, 4950; Pri 2260/7

(ma'ālì written wrongly), 2264/4, 2272/4, 2272/8, 2273

ذو الجلال و المقال (misspelling), SBB 4505

BSB 1735

(المعان) SBB 4944, Pri 2260/13 ذو الجلال وذو المعاني(!)

SBB 2408/4 ذو الجلال وذو الجول المعال

Pri 2260/8 ذو الجلال ذو المعال 
V. $18 \mathrm{a}$

: طر :

قهراً Gött., SBB 2407, 24082/2, 2408/3, 2408/4; 2408/5, 2408/6, 4496, 4505, 4944, 4950; Pri 2260/2, 2260/7, 2260/8, 2260/13, 2264/4, 2272/4, 2273; BSB 1735

V. $18 \mathrm{~b}$

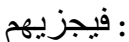

BSB 1735

V. 20b

: :

SBB 2408/4

V. 21a

: وينسو ن

فينسون Gött.; SBB 2407, 2408/3, 2408/4, 2408/5, 2408/6, 4496, 4505, 4944, 4950; Pri 2260/2, 2260/7, 2260/8, 2260/11, 2260/13, 2264/4, 2272/4, 2272/8, 2273; BSB 1735

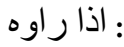

اذ اذ راوه (metrically impossible) Pri 2273

V. 21b

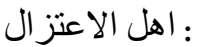

لا هل الاعتز ال ال ال ال 2264/4 (metrically impossible) Pri

V. 22a

: وما ان فحل

(metrically impossible) Pri 2260/8

(صلح :

omission in Pri 2273 (metrically and semantically impossible)

SBB 2408/4

ذو افتر اض (SBB 2408/4):

ذذا افتر اض دBB 4950; Pri 2260/13

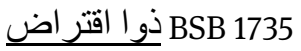

ذو افترض اضن (Gött., defective writing) 
V. 22b

: ذي التعالي

ذو التعال (grammatically incorrect) Pri 2260/11

BSB 1735 ذذا التعالي

V. 23a

: تصديق رسل

تصديق ارسل : تصديف (misspelling) Gött.

V. 23b

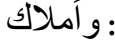

و إملك (wrong vocalization) Pri 2260/2

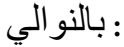

Gött.; SBB 2408/3, 2408/4, Pri 2260/2 (in the margin), 2260/7, 2260/13 (بالنو البوالئ), 2272/8

SBB 2407

V. $24 \mathrm{a}$

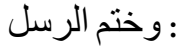

(wrong vocalisation) Gött.

ختم الرسل Pri 2272/8 (commentary)

V. 24b

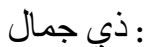

ذو ذي جلال دBB 24082/2

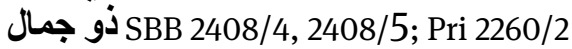

: نبي

Goett. (semantically impossible misspelling)

V. 25b

: و فيه

فقيه SBB 2407, 2408/3, 2408/4, 2408/5, 4496, 4944, 4950; Pri 2260/2, 2260/13, 2264/4, 2272/4, 2272/8; BSB 1735

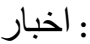

| BSB 1735 
V. $26 \mathrm{a}$

: بلا خلاف خلف

SBB 2407, 2408/2, 2408/3, 2408/5, 4496, 4505, 4944, 4950; Pri 2260/2, 2260/7, 2260/8, 2260/11, 2260/13, 2264/4, 2272/4, 2272/8, 2273; BSB 1735

V. $26 \mathrm{~b}$

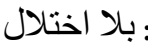

SBB 2408/4 بلا احتلال

V. 27b

الى يوم القيامة: (القيمة) in the reference manuscript) Gött. (metrically impossible)

القيّم (misspelling) Pri 2273

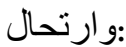

و اتحال (letter rā' missing) SBB 4944

V. 29a

: نبي

نبيّا (the better var.) Gött.; SBB 2407, 2408/3, 2408/4, 4496, 4505, 4950; Pri 2260/7, 2260/8, 2260/11, 2260/13, 2264/4, 2272/4, 2272/8, 2273

انثي

SBB 4496 (misspelling; semantically impossible)

V. $30 \mathrm{~b}$

لقمان:

SBB 2407, 4496, 4505, 4944, 4950; Pri 2260/8, 2260/11, 2272/8

: ماحذر :

فخزر (hearing mistake) Pri 2260/11

BSB Cod.arab. 1735

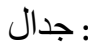

جذال (sem. imposs.) Pri 2273 
V. 31a

: يتوي

ينوي (misspelling) Pri 2260/13, BSB 1735

V. 31b

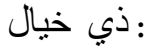

ذَي خبال ذött.; SBB 2407, 2408/2, 2408/3 (?, not clearly legible), 2408/4, 2408/5, 2408/6, 4496, 4950; Pri 2260/2, 2260/7, 2260/8, 2260/13, 2264/4; BSB 1735

V. 32a

كر امات

(singular) SBB 2408/4

V. 32b

و وهم:

فه Gött.; SBB 2407, 2408/2, 2408/3, 2408/4, 2408/5, 2408/6, 4496, 4505, 4944, 4950; Pri 2260/2, 2260/7, 2260/8, 2260/11, 2260/13, 2264/4, 2272/4, 2272/8, 2273; BSB 1735

V. 33a

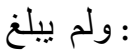

Gött., SBB 2407, 2408/2, 2408/3, 2408/4, 2408/5, 2408/6, 4496, 4505, 4950; Pri 2260/8, 2260/11, 2260/13, 2264/4, 2272/4, 2272/8, 2273; BSB 1735 يفصل (misspell.) Pri 2260/7

:ولي

grammatically incorrect) Pri 2260/11

V. 33b

التحال :

Gött. (metrically impossible)

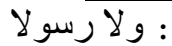

Pri 2260/2 او رسولا :

V. $34 \mathrm{~b}$

الإوفي : الاصحاب علي

(saut du même au même?) SBB 2408/6 علي الفاروق 
V. 35b

: عثمان :

Gött.

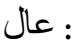

غال (semantically weak) Pri 2260/11

V. 36a

: وذو النورين

grammatically incorrect) SBB 2408/4, 2408/6; Pri 2260/2, 2260/11, 2260/13

ذذو النورين (metrically impossible) Gött.

V. $36 \mathrm{~b}$

: القتال

النقال (misspelling) Pri 2260/7

: في صف القتال

(semantically impossible) SBB 4944; Pri 2260/13; BSB 1735

V. 37b

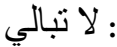

لا ابالي SBB 2408/5

V. 38a

: للصديقة :

BSB 1735

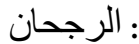

(metrically impossible) Pri 2260/11, BSB 1735

فاسمع

فُاعلم SBB 2408/3, 2408/4, 4505, 4950; Pri 2260/2, 2260/11, 2264/4 (comm.), 2272/4, 2272/8 (comm.); BSB 1735

V. $38 \mathrm{~b}$

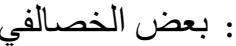

SBB 4944, 2408/2, 2408/3; Pri 2260/2, 2260/8, 2260/13, 2272/4, 2273

(grammatically imposs.) Pri 2272/8 (comm.) 
V. 39a

: بزيداً

يزيدَ (grammatically incorrect) Gött., BSB 1735

V. 39b

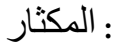

(misspelling) Pri 2272/8

: في الاغرا

(misspelling) SBB 2408/6 في الاغز

V. 41a

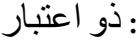

ذو العتبار (misspelling)

BSB 1735

V. 41b

: الانواع :

SBB 2408/3, 2408/4, 4496, 4505, 4944, 4950; Pri 2272/4, 2272/8 (comm.), 2260/11, 2260/2, 2260/7, 2260/8, 2260/13

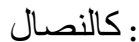

كا النصار Gött. (semantically weak; misspelling)

SSB 1735

كالفصال SBB 2408/4

V. 42a

: لذي :

بذي 2260/2

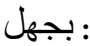

بحهل (dot missing, scribal error) Pri 2260/8

V. 42b

: بخلاق

لخلاق Pri 2260/2

لخلافSBB 2408/2 
V. $43 a$

ما ايمان:

إيمان (mā is missing, “omission”) SBB 2408/4, SBB 4944

V. $43 a$

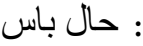

خال باس (misspelling) SBB 4505

B 2408/2, Pri 2264/4

V. $43 \mathrm{~b}$

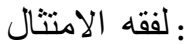

Gött., SBB 2407, SBB 2408/2, SBB 2408/3, SBB 2408/4, SBB 2408/5, SBB 4496, SBB 4505, SBB 4944, SBB 4950, Pri 2260/2, Pri 2260/11, Pri 2264/4, Pri 2272/4, Pri 2260/7, Pri 2260/8, Pri 2260/13

V. $44 \mathrm{a}$

حساب :

حسان (misspelling) Pri 2260/7

V. $44 \mathrm{~b}$

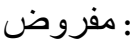

BSB 1735

V. $45 \mathrm{a}$

$\gamma_{g}:$

فُ Pri 2260/2

: : :

(metrically imposs.) Pri 2264/4

BSB 1735

V. $45 \mathrm{~b}$

: بعهر :

SBB 2408/2, SBB 4505, Pri 2260/7, Pri 2260/13

بعر SBB 2408/4, BSB 1735

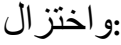

و واحتز ال (semantically impossible) SBB 2408/2, SBB 4944, SBB 4950, Pri 2260/7 
Gött. (semantically impossible)

V. $46 a$

ينوي:

ينو (defective writing) SBB 2408/3

Pri 2260/13

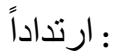

BSB 1735

V. 46b

يصير (metrically imposs.) Pri 2264/4, Pri 2272/4, Pri 2272/8 (com.), BSB 1735

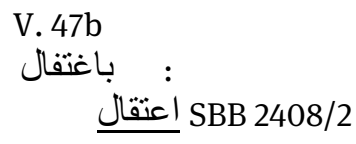

V. 48a

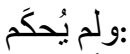

(metrically and sem. possible) Gött., B 2407, B 2408/2, SBB 2408/3, SBB 2408/4, SBB 2408/5, SBB 2408/6, SBB 4496, SBB 4505, SBB

4944, SBB 4950, Pri 2264/4, Pri 2272/4, Pri 2260/2, Pri 2260/7, Pri 2260/8, Pri 2260/13, BSB 1735

V. 48b

بما بـدي (also SBB 2408/6, Pri 2260/2):

بما يهذي (mockery - probably the better variant); Gött., SBB 2407, SBB 2408/2, SBB 2408/3, SBB 2408/4, SBB 2408/5, SBB 4496, SBB 4505, SBB 4950, Pri 2260/8, Pri 2260/11, Pri 2260/13, Pri 2272/4, BSB 1735

بما يهذر SBB 4944

بـ 2260/7

: و ويلغوا

SBB 2408/6, SBB 2408/2 ويلغو

ويلفط 2264/4 


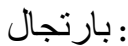

بار تحال (irtiḥāl, “departure” which is probably a misspelling for irtiǧāl) SBB 2407, Pri 2260/8, Pri 2264/4, BSB 1735

\section{49a}

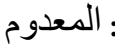

$$
\text { BSB } 1735
$$

V. 49b

ح':

$\dot{\tau}$ ( (misspelling, semantically impos.) Gött., Pri 2260/2

V. 50a

المكون: (الم:

المكو (misspelling) SBB 2408/6

V. 50b

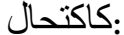

SBB 2407, SBB 2408/2, SBB 2408/3, SBB 2408/4, SBB 2408/5, SBB 2408/6, SBB 4496, SBB 4950, Pri 2260/7, Pri 2260/13, BSB 1735

V. 51a

لون جزء (misspelling):

\& كون جزان Gött., SBB 2408/3, SBB 2408/4, SBB 2408/5, SBB 2408/6, SBB 4496, SBB 4505, SBB 4944, SBB 4950, Pri 2260/11, Pri 2260/13, Pri 2272/4, Pri 2272/8, Pri 2273

V. 51b

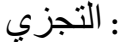

SBB 4950

SBB 2408/4:

Gött.

V. 52a

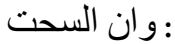

Gött. (semantically impossible, misspelling)

الصحت الص (misspelling) 


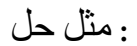

SBB 2408/5 دون شك

V. 52b

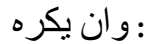

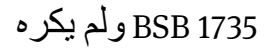

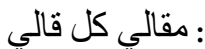

مقآل كل قال :defective writing) Gött., Pri 2260/7, Pri 2260/13

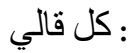

كل عل عل 2260/8

كل حل عال (misspelling; semantically impossible) SBB 4505

BSB 1735

V. 53a

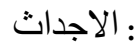

BSB 1735

V. 53b

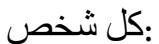

Sل علد (metrically and semantically possible) SBB 2408/2

V. $54 a$

بعضا:

SBB 2408/2, SBB 2408/3, SBB 4944, SBB 4950, Pri 2260/7; Pri 2260/13

SBB 4496, SBB 4505, Pri 2260/2, BSB 1735

V. 54b

: (من سوء الفعال

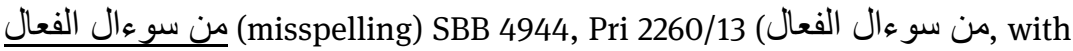
tashdìd fa“'āl)

B 2408/4 من شر الفعال

V. 55a

:

Gött. (expression haqq missing) 


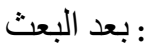

BSB cod.arab. 1735

V. $55 \mathrm{~b}$

: و وكونو ا بالتحرز

فكونوا Gött., SBB 2407, SBB 2408/2, SBB 2408/3, SBB 2408/5, SBB 4496, SBB 4505, SBB 4944, SBB 4950, Pri 2260/2, Pri 2260/7, Pri 2260/8, Pri 2260/11, Pri 2260/13, Pri 2272/8 (comm.), Pri 2264/4, BSB 1735

V. $56 \mathrm{a}$

فيعطى (also B 2408/2):

Gött.; SBB 2407, 2408/3, 4496, 4505, 4944, 4950; Pri 2272/8 (com.), 2260/2, 2260/7, 2260/8, 2260/11, 2260/13, 2264/4; BSB 1735

Gött. (vocalisation metrically impossible)

V. $56 \mathrm{~b}$

:وبعضا

BSB 1735

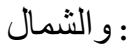

او الثمال (metrically impossible) Pri 2260/11

V. 57a

: وجري : و :

\& و و

V. 57b

: بلا احتمال

Gött.; SBB 2408/3, 2408/4, 2408/5, 4505, 4950, 4944 (dotting after letter $t \bar{a}^{\prime}$ of ihtibāl incomplete); Pri 2260/2, 2260/8, 2260/13, 2272/8 (comm.), 2264/4, 2260/7; BSB 1735

(in the margin the explanation بلا احتيال ) بلا اهتيال SBB 4496

SBB 2407

SBB 2408/2

Pri 2260/11 
V. 58b

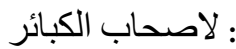

(metrically imposs.) Pri 2260/13

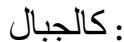

SBB 2408/4

V. 59b

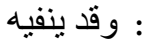

فقد ينفيه :SBB 4950

V. 60b

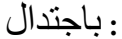

(the lectio optima: „Frohlocken“, i.e. “rejoicing”) SBB 2408/3, 2408/4, 2408/5; Pri 2260/8, 2272/4, 2272/8 (com.)

(metr. impos.) Gött.

باحتذ ال (misspelling, viz. wrong pointing: ḥā' instead of jīm) SBB 4944; Pri 2260/7, 2260/13, 2264/4

(wrong pointing: khāa' instead of jīm) SBB 4496; Pri 2260/11; BSB

1735

V. 61b

مر :

BSB 1735

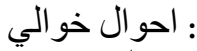

Gött. (misspelling)

I حوال حو ال SBB 2408/5, 2408/6; BSB 1735

both words lacking in SBB 4950

SBB 2408/2 ازئ خوان خوالي

احو ال خو ال حولي (khawālin, defective orthography) Pri 2260/13

V. 62a

: : :

Gött.

الجنان ( "gardens”, metrically and sem. poss.) Pri 2260/7, 2260/11

V. 63a

: و لا بفني 
SBB 2408/2 (feminine gender preferable)

SBB 2408/6 وما يفني

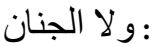

SBB 2407, Pri 2260/2, BSB 1735 ولا جنان

V. 63b

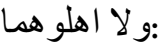

SBB 2407, SBB 2408/5, SBB 4496, SBB 4950, Pri 2260/2, Pri 2260/8, Pri 2264/4, Pri 2272/4, Pri 2273

Gött.

V. 64a

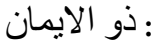

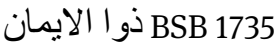

V. 64b

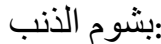

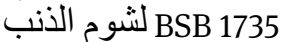

Gött.

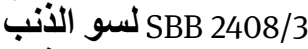

Pri 2260/13 بسوء الأنب

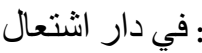

Gött.

SBB 2408/6, SBB 4496, SBB 4944, Pri 2272/8 في دار اشتخغال

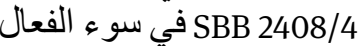

V. $65 \mathrm{a}$

نظم:

SBB 2408/4 التوحيد ونظيما(؟)

وشيا SBB 2408/2, Pri 2264/4

SBB 4496

V. 65b

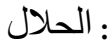

(misspelling; semantically impossible) SBB 4505 
V. 66a

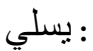

(metrically impossible) SBB 4496

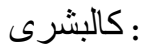

Sا 2260/13

V. 66b

: ويحي

SBB 2408/6

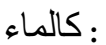

كا الماء Pri 2260/13

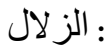

الذلال (misspelling) Pri 2260/11

V. 67a

: فخوضو :

(misspelling) Pri 2264/4

V. $67 \mathrm{~b}$

: تير اصناف المناف المنال

Gött., SBB 2407, SBB 2408/3, SBB 2408/6, SBB 4496, Pri 2260/2, Pri 2260/7, Pri 2260/8, Pri 2260/11, Pri 2260/13, Pri 2264/4, Pri 2272/8, BSB 1735

جنس اصناف المثال SBB 4505, SBB 2408/2

SBB 2408/4, SBB 2408/5, SBB 4944 حسن اصناف المنال

V. $68 \mathrm{~b}$

: في حال ابتهال

Gött. (grammatical mistake)

V. 69a

: بعفو الله بعفو :

ان يعفو BSB 1735 


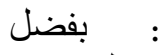

بفضله (metrically impossible) SBB 2408/5

V. 69b

(

يرزقه SBB 2408/4

V. 70a

: إن اله يجزي كل وقت

Gött., SBB 2408/2, SBB 2408/3, SBB 4505, SBB 4950, Pri 2272/4, Pri 2272/8, Pri 2260/8, Pri 2260/13

SBB 2408/4 وان الدهر ادعو كنها وسعي

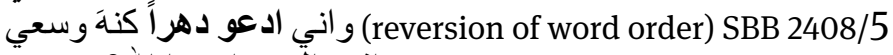

SBB 2408/6 واني الدهر ادعوا الله؟ وسعي الدي وني

Pri 2260/7 واني الدهر ادعو كل وقت الد الده

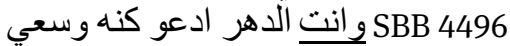

واني الحق ادعو كنه وسع SBB 2407 (in the margin correction, marked

by letter khä': kulla waqtin)

والحق ادعو كل وقت واني

BSB 1735 و اني الحق ادعو ا كل وقت كو الت

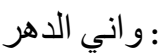

Pri 2260/11

V. 71b

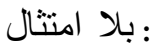

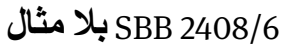

V. 72a

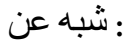

شبيه عن (metrically impossible) SBB 2408/6 


\subsection{Ibn al-Wardī, al-Lāmiyya al-Wardiyya}

The manuscript of reference is Petermann 8 (Ahlwardt no. 3999/3 = SBB 3999/3). Emphases set in bold indicate semantically and metrically possible variants. It may be worthy of note that there is a relatively high number of "real variants" in the manuscripts of Ibn al-Wardī's poem.

V.1a

SBB 3998, SBB 3999/6, SBB 3999/10:

الاغاني (,real variant“) SBB 3999/7, 3999/9, 3999/11, BSB 587, Baghdatlı Vehbi 1612, Esad Efendi 3690, 3507

و و الغزل SBB 3998, SBB 3999/6, 3999/7, 3999/10:

(misspelling, i.e. defective writing) BSB 1235

V. $1 \mathrm{~b}$

SBB 3999/3, 3998 :

SBB 3999/2, 3999/6, 3999/7, 3999/8, 3999/9, 4438, BSB 587, Esad

Efendi 3690

SBB 3999/5, Esad Efendi 3507

Baghdatll Vehbi 1612

V. 2a

SBB 3998:

SBB 3999/4, 3999/5, 3999/6, 3999/9, 3999/11, BSB 1235

SBB 3999/10

V. 3a

اهني :

SBB 3998, 3999/9, 4438, BSB 1235, Esad Efendi 3507, 3690

SBB 3999/1, 3999/4, 3999/5, 3999/8, 3999/10, 3999/11, BSB 587

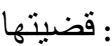

قصّيتها BSB 1235

V. $3 b$

ايامها :

SBB 3999/2, Baghdatlı Vehbi 1612 
V. $4 \mathrm{a}$

SBB 3999/7:

SBB 3998, 3999/1, 3999/4, 3999/5, 3999/8, 3999/9, BSB 587, 1235, Esad Efendi 3507, 3690

SBB 3999/1:

SBB 3998, 3999/3, 3999/7, 3999/8, 3999/9, 3999/10, 3999/11, 4438, BSB 587, 1235, Baghdatlı Vehbi 1612, Esad Efendi 3507, 3690

SBB 3999/4

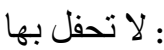

(metrically impossible) SBB 3999/4

V. $4 \mathrm{~b}$

تسمي (so?):

SBB 3998, 3999/1, 3999/2, 3999/4, 3999/5, 3999/7, 3999/8, 3999/9, 3999/11, 4438, BSB 1235

Esad Efendi 3507, 3690

BSB 587

V. $5 \mathrm{a}$

: واله

SBB 3999/10

SBB 4438 والة عن لهو الة و اله

BSB 587

V. $5 b$

: الامرد :

الاغيد BSB 1235

V. $6 a$

: تبدا

BSB 587, Esad Efendi 3690

V. $7 \mathrm{a}$

زاد:

فاق 3999/10 
اذ:

ان SBB 3999/2, 3999/7, 4438, Baghdatll Vehbi 1612

: بالنجم

SBB 3999/2, 3999/9, 3999/7, 3999/11, BSB 1235, Baghdatlı Vehbi 1612, Esad Efendi 3507, 3690

V. $7 b$

: بيدر :

مـSBB 3998, 3999/10, 3999/11

SBB 3999/2, 3999/7, Baghdatll Vehbi 1612

V. $8 \mathrm{~b}$

تجد :

ترى SBB 3999/5

نر ترن SBB 3999/9, BSB 1235

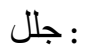

SBB 3999/4

SBB 3999/8

V.9a

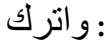

SBB 3998, 3999/1, 3999/2, 3999/5, 3999/7, 3999/8, 3999/9, 3999/11, 4438,

BSB 587, 1235, Baghdatlı Vehbi 1612

(misspelling) SBB 3999/10

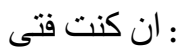

لا تحفل بها (error of the eye?) SBB 3999/5

V. $9 \mathrm{~b}$

: كيف :

SBB 3999/2

:

SBB 3999/7 
V. $10 \mathrm{a}$

:

و اتقى SBB 3998, 3999/1, 3999/2, 3999/5, 3999/7

فواتثSBB 3999/8, 3999/9, 3999/10, 4438, BSB 587, 1235

SBB 3999/6

V. $10 \mathrm{~b}$

: جاورت

صادفت SBB 3999/8

جاوزت (misspelling) BSB 1235

تاو لت

V. 11a

: يقطع :

SBB 3999/10 ينقطع

يهزم BSB 1235

: طر قا:

SBB 3999/4

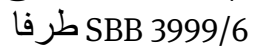

BSB 1235 جيشنا فرنا

بطلا : بطل

SBB 3999/6

V. $12 \mathrm{a}$

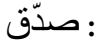

صدّ (letter qāf omitted) SBB 3999/1

V. $12 \mathrm{~b}$

: برصد : برصد

SBB 3999/6

: في الليل في

SBB 3999/2, 3999/7, 3999/8, 4438, BSB 587, Baghdatlı Vehbi 1612

: ملز : الز حل :

زخل 
V. 13a

: حارث :

حارة (orthographic mistake) SBB 3999/7, 4438

: مدرة

قدرت SBB 3999/1

SBB 3999/5 خدمة فرة SBB

V. $13 b$

: هدانا

هدنا (misspelling) SBB 3999/1

V. 14b

:

Sڤ SBB 3999/2, 3999/9, 4438, BSB 587

ذّل SBB 3999/8, 3999/11

: عرش :

SBB 3999/10, BSB 587, 1235

SBB 3999/2, 4438

ع sBB 3999/8, 3999/11

:

SBB 3999/4

V. $15 \mathrm{a}$

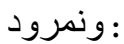

(substitution) SBB 3999/8, 3999/11 وقارون

: كنعان ونمرود :

نمرود وكنعان (inversion) SBB 3999/2, 3999/10, 4438

V. 15b : = v. 16b (error of the eye?) SBB 3999/8

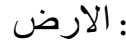

الامرد (mBB 3999/2, BSB 1235

(misspelling) SBB 3999/6 الارد 


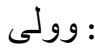

وو لا (orthographic mistake) SBB 3999/4, BSB 1235

V. $16 \mathrm{a}$

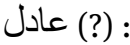

عاد SBB 3998, 3999/1, 3999/2, 3999/4, 3999/5, 3999/7, 3999/8, 3999/11, 4438, BSB 587, 1235

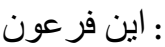

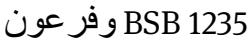

V. 16b :

= v. 15b, SBB 3999/8, 3999/9

V. 16b

: رفع :

قد بنا SBB 3999/4

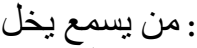

(cf. v. 4b: perhaps error of the eye) SBB 3999/7, 3999/11

V.17a

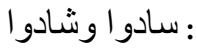

شادو ا وسادو ا و

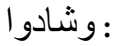

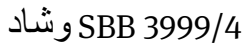

: وبنوا

ينو (misspelling?) SBB 3999/2

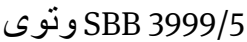

V. $17 \mathrm{~b}$

ا إل :

هلك SBB 3998, 3999/1, 3999/2, 3999/4, 3999/5, 3999/6, 3999/9, 3999/10, 3999/11, 4438, BSB 587, 1235

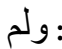

SBB 3999/10 


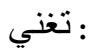

SBB 3998

تغن (the correct spelling) SBB 3999/1, BSB 1235

SBB 3999/6 يغني

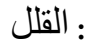

SBB 3999/8

V.18a

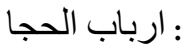

BSB 1235

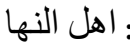

SBB 3999/10, BSB 587 اهل التقى الهل

V. $18 \mathrm{~b}$

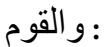

(misspelling?) SBB 3999/5 و القول

V.19a

إيعيد :

BSB 1235

كلا:

كلما (؟ SBB 3999/11

منهم:

منهمو (orthographic irregularity) SBB 3999/6

V.19b

:وسيجزي:

SBB 3999/2 وسيجز

ف فاعلا

فاعل SBB 3998, 3999/1, 3999/2, 3999/6, BSB 1235

V. 20a

- اي بني 
SBB 3999/10

(اسمع :

SBB 3998

V. 20b

: حكما

حكم (gramm. imposs.) SBB 3999/7

V. 21a

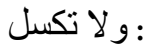

SBB 3999/6 فلا تكسل :ولا

V. 21b

: علي اهل : عل : عل : عل

عن اهل عل SBB 3998, 3999/4

V. 22a

: و احتفل

SBB 3999/8 فاحتفل

: للفقه

SBB 3999/5, BSB 1235

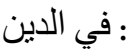

في الدنيا

V. $22 \mathrm{~b}$

: وخول

SBB 3999/4 و لا(؟) خول

V. 23a

: اربابه :

SBB 3999/6, 3999/11, BSB 1235

V. $24 \mathrm{a}$

:وحصلّل

فحصّله SBB 3999/1 


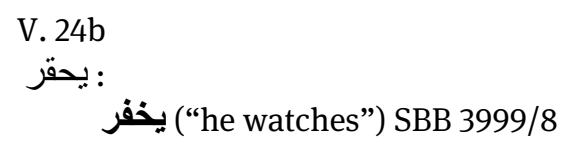

: بذل

SBB 3999/4 بدل

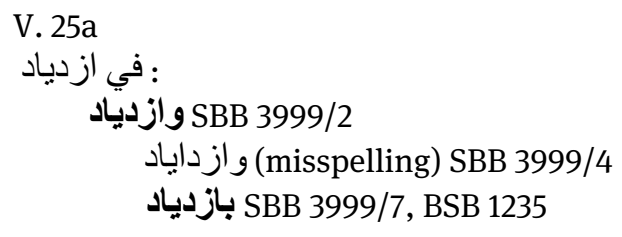

(ارغام

קBB 3999/11

: العدي : المدي

العدا SBB 3998, 3999/1

للعدا SBB 3999/11

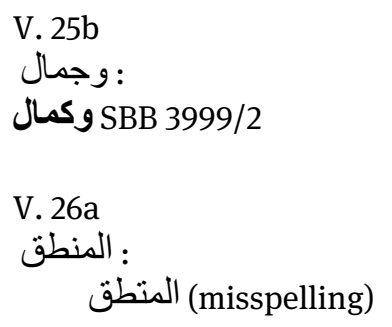

V. 26b

: يحرم :

SBB 3999/7 يخرم

BSB 1235

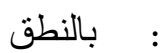

SBB 3998, 3999/1, 3999/4, 3999/5, 3999/6, 3999/8, 3999/10, 4438, BSB 587

V. 27a

: 
BSB 1235 وانظم

V. 27b

: في اطراح :

فاطراح ف فاطر SBB 3999/2, 3999/9, 3999/10, 4438

فا اطر اح (misspelling) BSB 587

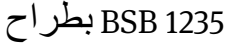

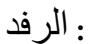

SBB 3999/5 الرقد

: بالدنبا؟

SBB 3999/4, 3999/6, 3999/7, 3999/8, 3999/10, 4438, BSB 587

: اقل :

SBB 4438

V. 28a

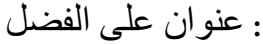

(omission) SBB 3999/5 عنوان الفضل على الفل

على : على :

عBB 3999/2

(omission) SBB 3999/5

: وما

فما SBB 3999/5, 3999/6

V. $28 \mathrm{~b}$

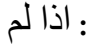

SBB 3999/4 اذ لم

: بيتذل

SBB 4438

V. $29 a$

: اهل الجود :

SBB 3999/2 اهل الخير

SBB 3999/8, 4438 اهل الفضل SBB 
V. 29b

: مقرف:

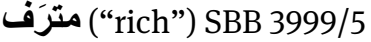

مقتر SBB 3999/7 (“miserly”)

مقر SBB 3999/6 مفرّ SBB

مفتقر (metrically impossible, semantically possible) BSB 1235

Baghdatlı Vehbi 1612

او من :

لمن BSB 1235

V. 30a

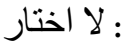

لاختار (misspelling) SBB 3999/11, BSB 1235

يدي (SBB 3999/5):

يدٍ (misspelling=unintentional semantic var.)

V. 30b

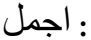

(misspelling) SBB 3999/5

V.31a

صرت : صر :

سرت SBB 3999/2

: عن مديحي :

SBB 3999/8

: مديحي

مديح SBB 3999/4

V. 31b

:و :

SBB 3998, 3999/1, 3999/4, 3999/5, 3999/6, 3999/8, 3999/9, 3999/10, 3999/11, 4438, BSB 587, 1235, Baghdatlı Vehbi 1612, Esad Efendi 3507

ال SBB 3999/7 
:

فتكفيني (grammatically incorrect) SBB 3999/2

SBB 4438 ينكفيني

V. 32a

اعذب :

اعدب (unpointed) Esad Efendi 3507

V. 32b

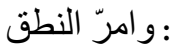

SBB 3999/1, 3999/2, 3999/4, 3999/5, 3999/6, 3999/7, 3999/9, 3999/10, 4438, BSB 587, 1235, Baghdatlı Vehbi 1612, Esad Efendi 3507

: بلعل

SBB 3999/4

V. 33a

: كسرى:

كسر (misspelling)

: تغني عنه

(omission of 'an) SBB 3999/5 تغني (عند)

عنه تغني (inversion) SBB 3999/9

مغني (omission of 'an) BSB 1235

V. 33b

اجز اء أجز (misspelling):

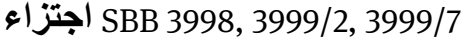

(اجترا) (BSB 1235, 587 (جترائ

SBB 3999/6, Baghdatlı Vehbi 1612

احتز أتصاص SBB 3999/8, 3999/11

| احتز SBB 4438

SBB 3999/1

Esad Efendi 3507

SBB 3999/9 ارتثافا

SBB 3999/10 اكتفاء SBB 
V. 35a

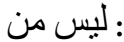

） ليس ما (the better var., semantically) SBB 3998, 3999/1, 3999/2, 3999/4, 3999/5, 3999/6, 3999/7, 3999/8, 3999/9, 3999/11, 4438, BSB 587, 1235, Esad Efendi 3507

: بحوي الفتى

SBB 3999/8 يخوي الفتى

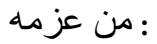

عBB 3999/8, 3999/11, BSB 587

Baghdatli Vehbi 1612

V. 35b

: يوما :

منـ SBB 3999/5, SBB 3999/8, 3999/11, BSB 1235

V. 36a

: قاطع

حاطر SBB 3999/2

SBB 4438

فواتطرSSBB 3999/7, Baghdatlı Vehbi 1612

فاقطع SBB 3999/6, 3999/10

: مار : عار اتها

SBB 3998, 3999/1, 3999/2, 3999/4, 3999/5, 3999/6, 3999/7, 3999/8, 3999/9, 3999/10, 4438, BSB 587, 1235, Baghdatlı Vehbi 1612, Esad Efendi 3507

V. 36b

(misspelling) SBB 3999/4, 3999/6

:تعلي : تعلي

SBB 3999/10, BSB 587, 1235

V. 37a

: الر اغد :

(الزاهد (perhaps the better var.) SBB 3998, 3999/1, 3999/4, 3999/5, 3999/6, 
3999/9, BSB 587, 1235, Esad Efendi 3507

: تحصيلها

(misspelling) SBB 3999/4 تحصلها

V. 37b

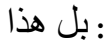

SBB 3999/4 يا هذا بل

: - ماذل

SBB 3998

ذل ذل (omission of the initial letter) SBB 3999/4

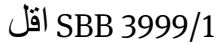

V. 38a

: مُنْرِ مُكْنْرُ

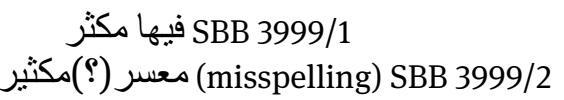

V. $38 \mathrm{~b}$

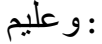

(misspelling) SBB 3999/2

BSB 1235

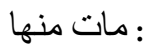

مات فيهات مات من مB 3999/8

مات منه فئه

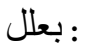

بغلل (misspelling) SBB 3998

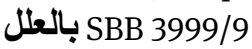

V. 39a

: منها :

فيها SBB 3999/1, 3999/4, 3999/5, 3999/8, 3999/11, BSB 587, Esad Efendi 3507

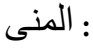

منى 3BB 3999/5, 3999/9, Esad Efendi 3507

BSB 1235 
V. 39b

: غابات :

غيات (misspelling) SBB 3999/4

V. 40a

فاتر : فاتر :

SBB 3999/2, BSB 1235

SBB 3999/8

: و اتئد

و وايتئد

SBB 3999/2

SBB 3999/8, 3999/11 تهندي

SBB 4438

V. 41a

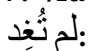

لا

لا لغ ت 1235

مol:

مهمـ SBB 3998, SBB 3999/5, 3999/8, Baghdatlı Vehbi 1612 (؟) سهوماً SBB 3999/6

: تُفَدْ

يفد (without diacritical dots) BSB 1235

اله :

SBB 3999/8

منه :

SBB 3999/7

V. 41b

: فرماها

Esad Efendi 3507 
V. 42a

ابدا :

ابدَ (misspelling) SBB 3999/4

V. $42 \mathrm{~b}$

اصل :

SBB 3999/4

ما قد : ما

L SBB 3998

: حصل :

عBB 3999/1

V. 43a

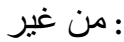

عن غير SBB 3998, 3999/1

V. $44 \mathrm{a}$

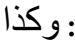

انما Esad Efendi 3507

: الثو :

لشوك (misspelling) SBB 3999/4

SBB 3999/8

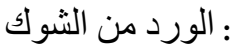

(inversion of word order) SBB 3998, 3999/5 الشوك من الورد الورد

V. $44 \mathrm{~b}$

: يطلع :

SBB 3999/7, 3999/8, 3999/9, 3999/11, 4438, Esad Efendi 3507

BSB 587

بصل : بصل

يصل (misspelling) SBB 3999/4 
V. 45a

SBB 3999/10:

SBB 3999/2 غير اني

V. 45b

: - و اذ بابي بكر

(ذاذ بي يكر : (sic? misspelling) SBB 3999/6

: اتصل :

SBB 3998, 3999/1, 3999/5, BSB 587, 1235

V. $46 a$

ق قيمة

قيم SBB 3999/2

V. 46b

و او او آَقَل

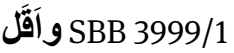

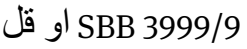

V. 47a

كتم:

SBB 3999/8

SBB 3999/8, 4438 (?) :

فقراً وغنيً SBB 3998, 3999/2, 3999/4, 3999/5, 3999/6, 3999/10, 3999/11,

BSB 587, 1235

V. $47 \mathrm{~b}$

: : : :

Esad Efendi 3507

V. $48 \mathrm{a}$

و ادّّ (omission of letter $r \bar{a})$ :

و وادرع SBB 3998, 3999/1, 3999/2, 3999/6, 3999/7, 3999/8, 3999/9, 3999/10, BSB 587,1235, Esad Efendi 3507 
كداً وجداً SBB 3999/11

جدّاً وكدّاً (inversion of word order) SBB 3998, 3999/2, 3999/6, 3999/9, 3999/10, 4438, BSB 587

V. 48b

: الحمقى :

SBB 3999/2

(metrically impossible) SBB 3999/8

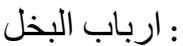

SBB 3999/10

SBB S438 - S ارباب الخلل SBB

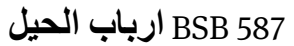

V. 49a

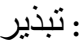

تبدير (misspelling) B 9998

تدبير (misspelling) SBB 3999/1, 3999/2, BSB 1235

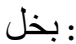

(misspelling) SBB 3999/4

وفتر (error of the eye) SBB 3999/6

V. 49b

: و كلا

فكلا SBB 3998, 3999/1, 3999/2, 3999/4, 3999/8, 3999/9, 3999/11, 4438, BSB 587, 1235, Esad Efendi 3507

كل من (metrically impossible) SBB 3999/5

فكن 35B 399/6

V. 50a

SBB 3999/5 (metrically impossible):

لا تخض (semantically better) SBB 3999/1, 3999/2, 3999/4, 3999/7, 3999/8, 3999/9, 3999/10, 3999/11, 4438, BSB 587, Esad Efendi 3507

BSB 1235

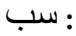

SBB 3999/5 
SBB 3999/8, 3999/11

سادات :

SBB 3999/1, 3999/4 مادة ذاب سناد SBB 3999/5

SBB 3999/5 ما ذات

: مضوا :

مضت SBB 3999/2

V. $50 \mathrm{~b}$

انه :

فهم BSB 1235

V. 51b

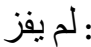

لم لم بفذ (misspelling) SBB 3999/10

SBB 4438

لمن لمن 1235

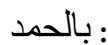

بالجد 39B 399/4

BSB 1235

SBB 3999/8

عقل : عقل :

غفل SBB 3998, 3999/1, 3999/4, 3999/5, 3999/6, 3999/7, 3999/8, 3999/9, 3999/10, Esad Efendi 3507

عفل (misspelling [defective pointing]) BSB 1235

V. 52a

: من ضد : مند :

SBB 3999/8, 4438

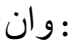

SBB 3999/5, 3999/7, 3999/9, Esad Efendi 3507 ولو

V. 52b

: حاول :

BSB 1235 


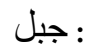

SBB 3999/1, 3999/2, 3999/4, 3999/9, BSB 587

V. $53 a$

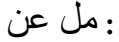

(بعد SBB 3999/8, 3999/11

SBB 3999/10

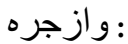

SBB 3999/8 واحذره

SBB 3999/10, 4438, Esad Efendi 3507

V. $54 a$

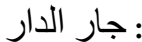

SBB 3999/2, 3999/7 جار السوء

SBB 3999/8 - S - S جار الجنب

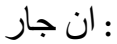

SBB 3999/5 لو جار SB S

جار (omission of a word) SBB 4438

V. 55a

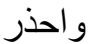

عsad Efendi 3507

V. 55b

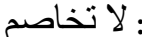

لا تخصم (omission of a letter) SBB 3999/2

V. 56a

:وان هم

SBB 3999/4 و انهم

: تلي

BSB 1235 تل 
: عدلو :

سالوا SBB 3998, 3999/1, 3999/4, 3999/5, 3999/6, 3999/7, 3999/8, 3999/9, 3999/10, 3999/11, 4438, BSB 587, 1235

V. 56b

: عدل :

غذل SBB 3999/1

عذل (the better var.) SBB 3999/2, 3999/6, 3999/8, 3999/9, 3999/10, 3999/11, BSB 587, 1235

عزل (misspelling(?)) SBB 4438

رغبة فيكة :

(dittography) SBB 3999/5 رغبة فيك رغبة :رغ فر

V. 57a

اعداء : عاء :

اعذاء (misspelling) SBB 3999/6

V. 57b

:

SBB 3999/2

SBB 3999/8

V. 58a

:و هو :

فه SBB 3998, 3999/1, 3999/2, 3999/4, 3999/6, 3999/8, 3999/9, 3999/10, 4438, BSB 587, 1235, Esad Efendi 3507

: عن لذته

SBB 3999/2

كو :

فكلا SBB 3999/8

V. 59a

:للقصص:

SBB 3999/8, BSB 1235

للنقض (naqd = misspelling for naqs) SBB 3999/2 


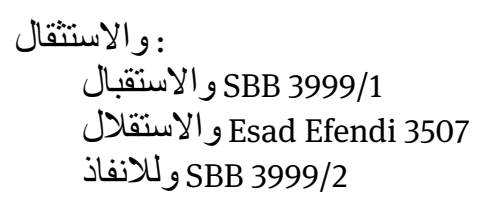

V. 59b

: لفظة

لفضة (misspelling) SBB 3999/1

: لو عظاً

SBB 3998, 3999/5, 4438, BSB 1235

و و عظًاً SBB 3999/4

V. 60a

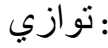

SBB 3999/1

SBB 3999/8, 3999/11 توازن SBB

لما:

بما SBB 3998, 3999/1, 3999/2, 3999/6, 3999/8, 3999/9, 3999/10, 3999/11,

BSB 587, 1235, Esad Efendi 3507

ما (metrically impossible) SBB 3999/4

بها (semantically and grammatically impossible) SBB 3999/5

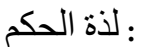

لذة العزل (error of the eye) SBB 3998, 3999/2

V. $60 \mathrm{~b}$

ذاقه :

ذاقها SBB 3999/8, 3999/10

: الثخص :

(metrically and semantically possible) SBB 3999/4

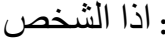

SBB 3999/6

V. 61a

: تو الو لايات 
فالولايات (misspelling) SBB 3999/5

فالو لايات SBB 3999/7, 3999/8, 3999/10, 4438, BSB 587

: و إن طالت

SBB 3999/1, 3999/2, 3999/4, 3999/6, 3999/7, 3999/10, 3999/11, 4438, BSB 587, 1235

ذاك:

ذلك (metrically impossible) SBB 3999/4

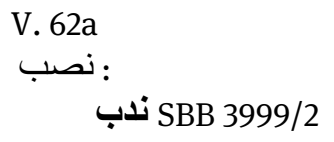

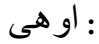

BSB 1235

V. 62b

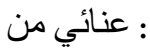

SBB 3999/2 عنداد

SBB 3999/9

عناني عن (misspelling) BSB 1235

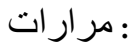

Sم مرار SBB 3998

مداراة (the better variant) SBB 3999/2, 3999/10, BSB 587

مدار مدات SBB 3999/4, 3999/5, 3999/6, 3999/7, 3999/8, 3999/9, 3999/11, 4438,

Esad Efendi 3507

V. 63a

تفز

تفذ (misspelling) SBB 3999/10

V. 63b

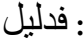

SBB 3999/4 في دليل

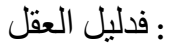

SBB 3999/7 
SBB 3999/6 فدليل الفضل

V. 64a

ان

و وان SBB 3999/2

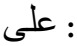

عن BSB 1235

V. $64 \mathrm{~b}$

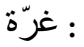

عزة (misspelling) SBB 3999/1, BSB 1235 غرت (misspelling) BSB 587

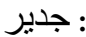

جدير SBB 4438

جديد Esad Efendi 3507

V. 65a

- ت

زور (misspelling) SBB 3999/2

تز

تزدد (metrically impossible) SBB 3999/2

V. $65 \mathrm{~b}$

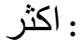

SBB 3999/2 يكثر SBB

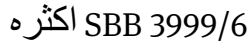

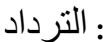

(misspelling) SBB 3999/4 الترداذ

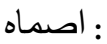

(ضماه اضماه

SBB 3999/8, 3999/9

اطناه (hearing mistake for aḍnāhu?) SBB 3999/2

اعياه SBB 3999/7

اخماه (semantically impossible) SBB 3999/6 
V. 66a

: بنصل السنيف :

بفعل السيف :metrically possible but semantically weak) SBB 3998, 3999/1, $3999 / 5$,

3999/6, 3999/9

SBB 3999/7, 4438, BSB 587

V. $66 \mathrm{~b}$

واعتبر

Esad Efendi 3507

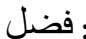

فصل (misspelling) BSB 587

:

SBB 3999/7

Esad Efendi 3507

: الحُلَل

(misspelling?) SBB 3999/2, 3999/8, 3999/9, 4438

الملل (error of the eye) SBB 3999/6

V. 67a

: النصل

الفضل (better variant) SBB 3998, 3999/1, 3999/4, 3999/9, 3999/10, 3999/11, 4438,

BSB 587, 1235

(dittography) SBB 3999/8

SBB 3999/7 الطفل

(semantically and metrically possible) SBB 3999/5

:

SBB 3998, 3999/1, 3999/7, 3999/8, 3999/9, 3999/10, 3999/11, 4438, BSB 587, 1235, text ed. A. Raux, Esad Efendi 3507 SBB 3999/6 اطلن 
V. $68 \mathrm{a}$

: عجز ظاهر :

SBB 3999/6 عجز أ ظاهراً فجر

SBB 3999/8 عجز فُاغتنم

V. 68b

: فاغترب :

SBB 3999/8

فاعترب (misspelling?) SBB 3999/10

: تلقي

تلق (defective script) SBB 3998, 3999/2, 3999/5, 3999/9, Esad Efendi 3507 نلتقي (metrically impossible) SBB 3999/4

V. 69a

: فبمكث

فمكث: (syntactically incorrect) BSB 587

: بيقى :

BSB 1235

آسنا

(misspelling) SBB 3999/2

V. 69b

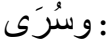

SBB 3999/4

سر (omission of a letter) SBB 4438

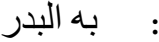

البدر (omission of a word) SBB 3999/4

V. 70a

العائب

Esad Efendi 3507

عبثاً :

عابثا SBB 3999/1, 3999/4, 3999/7, 3999/8, 3999/9, BSB 587

عايباً SBB 3998, 4438 
عامدا SBB 3999/5, BSB 1235

عاتبا SBB 3999/6, Esad Efendi 3507

سفهاً: SBB 3999/10

V. $70 \mathrm{~b}$

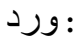

BSB 1235

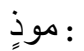

(misspelling for $y u$ 'dhî?) SBB 3999/10, BSB 587

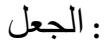

BSB 1235 الجفل(؟)

V.71a

: عدّ :

غُ BSB 1235

: لفظي

SBB 3999/2, 3999/10

نظمي

SBB 4438

: - واستتر

SBB 3999/2

BSB 1235

V.71b

تُعل

ثقل (misspelling ?) SBB 3999/5, 3999/8

شعل (MBB 4438

V.72b

: لين

لينا (metrically and grammatically possible) SBB 3999/5, 3999/7, 3999/8, 3999/10, BSB 587, 1235

: ان ان الحيات الخ

this hemistich comes as v. 71b in SBB 3999/6 
V. $73 a$

il :

رب SBB 3999/5

V. 73b

: و واذا

Sمتى SBB 3998, 3999/2, 3999/4, 3999/6, 3999/7, 3999/9, 3999/10, 4438, BSB 587, Esad Efendi 3507

مخن : مخن :

(misspelling) SBB 3999/4 سجن

SBB 3999/6 يسخن

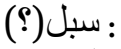

(semantically weak: “to be courageous”) SBB 3999/5, 3999/9, 3999/11, BSB 587

قتل SBB 3999/10, 4438, BSB 1235, Raux’s ed.

SBB 3999/4

V.74a

ان

SBB 3999/5 (ودت or ورت (or)

كالخيروز (misspelling, possibly due to Persian influence), SBB 3999/4, Esad Efendi 3507:

كالخيزور ("bamboo”, correct) SBB 3998, BSB 587

(metrically impossible) SBB 3999/5 كالخيزران (misspelling) BSB

كالخيرور (misspelling) BSB 1235

V. 74b

: لين :

لان SBB 3998, 3999/1, 3999/2, 3999/4, 3999/6, 3999/10, BSB 587, 1235,

Esad Efendi 3507

: شُئت

BSB 1235

: انفتل : 


\section{SBB 3999/1, BSB 1235 انتقل \\ SBB 3999/8 فتل SBB \\ SBB 3999/5, 4438 اعتل فتل}

V. 75a

ز مان

زمن (metrically impossible) SBB 3999/4

ز زماني 39B 3999/6

is:

: (semantically impossible) SBB 3999/6

V. 75b

ه :

SBB 3999/4

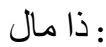

ذوو مال (grammatically impossible) SBB 3999/2

SBB 3999/10

V. $76 a$

و اجب عند :

و اجب عب عند (misspelling) SBB 3999/1

: الورى :

BSB 1235

V. 76b

-

SBB 3998, 3999/1, 3999/4, 3999/6, 3999/7, 3999/8, 3999/9, 3999/10, 3999/11 (يستقال?), 4438, BSB 587, 1235

(metrically impossible) SBB 3999/5

V. 77a

غمّر : عمر :

عمر SBB 3999/1, 3999/6

(metrically and semantically possible) SBB 3999/5, 3999/7 
V. 77b

منهو (irregular orthography), BSB 587:

منهم (the correct spelling) SBB 3998, 3999/1, 3999/7, 4438, BSB 1235, Esad Efendi 3507, 3690

كاتر :

BSB 1235

V. 79a

: وصلوة

(irregular orthography) SBB 3999/5 وصلو ات

V. 79a-b

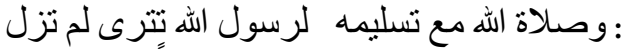

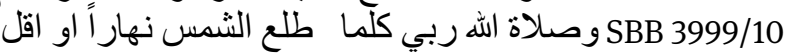

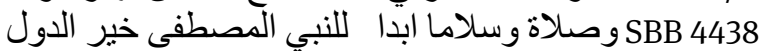

V. $80 \mathrm{~b}$

ط :

(dittography of $m \bar{a})$ SBB 3999/5

\section{4 -Laqānī, Jawharat al-tawḥīd}

The manuscript of reference is SBB-PK, Hs. or. 4831.

v. $2 \mathrm{~b}$

خلا Spr. 1956; printed edition:

Ms. or. 618

V. $4 \mathrm{a}$

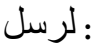

رسل Pm. 703

V. $4 \mathrm{~b}$

: وصحبه وحزبه

Spr. 1953 من بعده وصحبه وصحبه

v. $8 \mathrm{a}$

: في القبول 
بالقبول Pm. 703

v. $9 \mathrm{~b}$

بو.Hs. or. 4831:

Spr. 1956, Pm. 703, Spr. 1953

و جبا print:

Ms. or. 618

v. $11 \mathrm{~b}$

Hs. or. 4831:

Spr. 1956, Pm. 703, Spr. 1953 يخل من

v. $15 a$

Hs. or. 4831:

Spr. 1956

v. $15 \mathrm{~b}$

Hs. or. 4831:

Spr. 1956 الى العالم

print:

Ms. or. 618

v. $17 \mathrm{a}$

: وكل ما

Pm. 703 وكلما

V. $19 \mathrm{~b}$

: شطر

ثر ط Pm. 703

v. $20 \mathrm{~b}$

: مادر

قادر We. 1732

v. $21 \mathrm{~b}$

: تزيد

يزيد We. 1732 
v. $25 \mathrm{a}$

قيامه :

(wa-in wa-qiyāmuhū crossed out) Pm. 703

v. $26 \mathrm{a}$

: شربك :

We. 1732

v. $28 \mathrm{~b}$

: سبيل الحق :

طريق الحق Pm. 703, We. 1732

v. $29 \mathrm{a}$

حياته :

Pm. 703

v. $29 \mathrm{~b}$

لذا : لذا بذي Pm. 703

We. 1732

V. $31 \mathrm{~b}$

سمع :

Spr. 1953, 1956, Pm. 703, We. 1732

v. $32 \mathrm{~b}$

print; Ms. or. 618, We. 1732, Spr. 1953

Hs. or. 4831

v. $33 \mathrm{a}$

: فقدرة :

Spr. 1956 وقدرة

v. $34 \mathrm{~b}$

لكن عم ذي print; Spr. 1956, Pm. 703, We. 1732

Hs. or. 4831

v. $35 \mathrm{a}$

و الممتنعا (print): و الممتنع Ms. or. 618, Spr. 1953, 1956 
Pm. 703

V. $36 \mathrm{a}$

print; Ms. or. 618, Pm. 703, Spr. 1953:

Hs. or. 4831

We. 1732

v. $38 \mathrm{a}$

: عظيمة :

Spr. 1953, 1956, Pm. 703, We. 1732

v. $41 \mathrm{a}$

كائ كلامه :

We. 1732

v. $42 \mathrm{a}$

: -

We. 1732

v. $44 \mathrm{a}$

: ما امكنا

We. 1732

v. $44 \mathrm{~b}$

:

Spr. 1956, Pm. 703

V. $45 \mathrm{~b}$

: موفق

We. 1732

v. $46 a$

: بعده :

بعبده (misspelling) We. 1732

v. $47 a$

: -

We. 1732 
v. $48 \mathrm{a}$

كلف:

Spr. 1953, 1956, Pm. 703, We. 1732

v. $48 \mathrm{~b}$

Pm. 703

: مؤثر فلتعرفا

Spr. 1953, 1956, Pm. 703, We. 1732

v. $50 \mathrm{a}$

يثبنا print:

Hs. or. 4831

Spr. 1953, 1956, Pm. 703, We. 1732

v. $53 a$

:

We. 1732

v. $53 \mathrm{~b}$

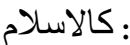

كاسلام Pm. 703

v. $57 \mathrm{a}$

جمع print :

Hs. or. 4831, Spr. 1953, 1956, Pm. 703, We. 1732

v. $57 \mathrm{~b}$

فن print; Pm. 703, We. 1732, Spr. 1953:

بs. or. 4831

v. $58 \mathrm{~b}$

: هوى :

هe. 1732

v. $59 a$

الامانة

امانة Pm. 703 
v. 60b

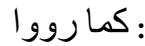

كما روو - مكارو

v. $62 \mathrm{a}$

: شهادت :

شهادة We. 1732

v. $62 \mathrm{~b}$

شهادتا print; Spr. 1953, 1956:

شهادت Hs. or. 4831

شهادة Pm. 703

v. $63 \mathrm{~b}$

: رقي في الخير

رقي الخير (om. of fi) We. 1732

v. $64 \mathrm{a}$

print, We. 1732, Spr. 1953:

Hs. or. 4831

v. $66 \mathrm{~b}$

:

Spr. 1953 ذوو الفضل

v. 67a

: فصلو ا اذ فضلو ا

We. 1732

v. $67 \mathrm{~b}$

وبعض كل

We. 1732

: يفضله

Spr. 1956 يفضلو ا

We. 1732, Spr. 1953 
v. $70 b$

: حتى الزمان

على الزمان Pm. 703

v. $72 b$

: من غض :

من عض عن (spelling mistake) Pm. 703

من غمض من (misspelling) We. 1732

v. $74 \mathrm{a}$

رووا:

روو We. 1732

v. $74 \mathrm{~b}$

print, Pm. 703, We. 1732, Spr. 1953:

Hs. or. 4831

V. $75 b$

فتابعي فتابع print, Spr. 1953, 1956, Pm. 703, We. 1732:

Hs. or. 4831

v. $76 \mathrm{a}$

و وخير ه لمن ولي :

Hs. or. 4831, We. 1732, Spr. 1953, 1956, Pm. 703

V. $76 \mathrm{~b}$

: - و امر هم

فامر هم Pm. 703

V. $78 \mathrm{a}$

: العظيم الشنان

هo 703

We. 1732

V. $78 \mathrm{~b}$

فبيعة print, Spr. 1953, 1956, Pm. 703:

Hs. or. 4831, We. 1732 
v. $82 \mathrm{a}$

: خبر :

Spr. 1956, Pm. 703

v. $83 a$

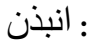

انبذاً (spelling mistake) Spr. 1956

V. $85 \mathrm{a}$

عبد :

عند misspelling, Hs. or. 4831

v. $85 \mathrm{~b}$

: خيرة

We. 1732

v. $86 a$

شُينًا

سيّئيا (? misspelling) Hs. or. 4831

We. 1732

V. $86 \mathrm{~b}$

:

We. 1732

v. $87 a$

قلل الاملا print, Hs. or. 4831:

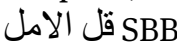

قلل املا

Spr. 1953, 1956, We. 1732 وقل الاملا

v. $88 \mathrm{~b}$

: وبقبض

Spr. 1953 وبقبض

v. $90 \mathrm{a}$

: لدى النفخ

Spr. 1956 
V. $90 \mathrm{~b}$

: السبكي

Spr. 1956

: اللذ :

كذا We. 1732

v. $91 \mathrm{~b}$

:ووضحا

We 1732

v. $92 \mathrm{a}$

خصوا : حصصو :

خصو Pm. 703

v. $93 a$

نخض print :

SBB

v. $94 \mathrm{a}$

هو هي Hs. or. 4831:

هي We 1732, Ms. or. 618, Pm. 703, print

v. $95 \mathrm{a}$

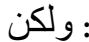

Spr. 1953

:

قد رما Pm. 703

v. $95 \mathrm{~b}$

خلافا

خلاف Pm. 703

ما فسروا print:

بما قد فسرو : Spr. 1953 (added in the margin) 
V. $96 \mathrm{~b}$

: واجب

اوجب Pm. 703, Spr. 1953

v. $98 \mathrm{a}$

: لكن ذا :

Spr. 1956

V. $99 \mathrm{~b}$

رُجِّحَت

رجّحة

V. $100 \mathrm{~b}$

: وما في حق ارتياب

(the text has been changed at this place) Pm. 703

V. 101a

فالسيّئات print, Ms. or. 618, Spr. 1953, 1956, Pm. 703:

We 1732

Hs. or. 4831

v. $102 a$

print, Ms. or. 618, Spr. 1953, 1956, Pm. 703, We 1732:

Hs. or. 4831

V. $103 \mathrm{~b}$

: فخفف :

Spr.1956 حفّه

v. $104 \mathrm{~b}$

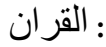

Spr. 1953 Sp S S

v. $105 \mathrm{~b}$

و او الاعيان print, Ms. or. 618, Spr. 1953, 1956, Pm. 703:

Hs. or. 4831

We 1732 
V. $106 \mathrm{~b}$

: ومنتلف

Spr. 1953 و منلف

V. $107 a$

: م العرش

We 1732

V. $107 \mathrm{~b}$

: ت الكاتبون

We 1732

v. $110 \mathrm{a}$

دار print, Ms. or. 618, Spr. 1956, We 1732:

Hs. or. 4831, Spr. 1953

Pm. 703

V. $112 \mathrm{~b}$

: بعهدهم

We 1732

: قلّ :

Spr. 1956

يز print :

Ms. or. 618, Spr. 1956

يظ ذو (hearing mistake?) We 1732

v. $114 \mathrm{~b}$

لما قد print:

كما قد Ms. or. 618, Spr. 1953, 1956, We 1732

print, Ms. or. 618, Spr. 1953, 1956:

Hs. or. 4831

v. $115 \mathrm{~b}$

ע print:

فلا Ms. or. 618, Spr. 1956, We 1732 
print:

Spr. 1956

v. $118 \mathrm{a}$

بالحياة print :

Hs. or. 4831

V. $118 \mathrm{~b}$

مشتهى print :

مشتها Hs. or. 4831

V. $119 a$

: عند القوم ما بـ : مابه

ما بـ (omission of words) Spr. 1956

v. $123 a$

eprint :

عنه Hs. or. 4831

V. $124 \mathrm{~b}$

: كبيرة :

Spr. 1953 وكبيرة

:

We 1732

V. $125 \mathrm{~b}$

: ولا انتقاض

Spr. 1956 و الانتقاض

V. $127 \mathrm{a}$

مال نصب print :

مال نسب :Ms. or. 618, Spr. 1953, 1956

ما لا نسب فل مالب 1732

V. $129 a$

: نفى :

نفا Spr. 1953 
V. $132 \mathrm{~b}$

(ذاه : -

اذا We 1732

v. $133 \mathrm{~b}$

ان ان ازيل

Ms. or. 618

Spr. 1956, We 1732

V. $135 \mathrm{a}$

كالعجب print:

كالعجب و الكبر Ms. or. 618, Spr. 1953, 1956, We 1732

V. $135 \mathrm{~b}$

:

Spr. 1953, 1956

We 1732

V. $136 \mathrm{a}$

(و ولكن print :

وكن We 1732, Spr. 1953

Hs. or. 4831, print:

حيار Hs. or. 4831

V. $138 \mathrm{~b}$

: ابيح :

Spr. 1956

v. $139 \mathrm{a}$

: الصالح :

(writing mistake) Spr. 1956

V. $139 \mathrm{~b}$

مدا اخلفا Hs. or. 4831 :

print, Ms. or. 618, We 1732, Spr. 1953, 1956

V. $140 \mathrm{~b}$

print : 
Hs. or. 4831

V. $141 \mathrm{a}$

print:

print; Ms. or. 618, We 1732, Spr. 1953

V. $141 \mathrm{~b}$

Hs. or. 4831 :

print, Ms. or. 618, We 1732, Spr. 1953

Spr. 1956 ومن يمل فمن

Ms. or. 618, print :

غو Hs. or. 4831

v. $143 \mathrm{~b}$

: دابه

ذابه (spelling mistake) We 1732

v. $144 a$

و و و Hs. or. 4831 :

و اله print, Ms. or. 618, Spr. 1953 


\section{5 -Sanūsī, al-'Aqīda al-ṣughrā}

The reference text is the edition of M. Wolff. Substantial, i.e. semantically possible variants have been highlighted by use of bold script. In the notes added to some selected variants an attempt has been made to characterize the type of variance it represents and to identify the cause of its occurrence (for an evaluation

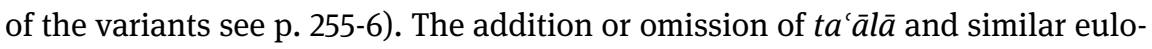
gies following the word Allah or the various expressions used in praise of Muhammad and other "prophets" have not been registered for inclusion in this list of textual variants.

p. 2, 1.3 (ed. Wolff)

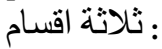

(grammatical mistake: transgression against the rule of gender polarity) We. 1685

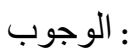

(a different word; copyist mistake) Schöm.

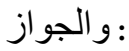

(different word; copyist mistake) Dq. 97

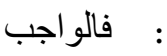

فالوجوب (different word; copyist mistake) We. 1793

1.4

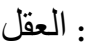

(copyist mistake involving addition of letter yā') Dq. 97

ما

مل (orthographic mistake) Schöm.

1.5

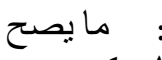

(different word; a substantial variant) Dq. 97

: : :

او عدمه (copyist mistake) Pe. 105 
1. 6-7

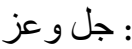

(substantial variant - inversion of word order) We. 1685

1. 7<smiles>[AsH2][AsH2]</smiles>

omitted and supplied in the margin, We. 1793

1. 8

: فما بجبب :

فمما يجب (copyist mistake, albeit semantically possible) We. 1685, 1793

1.9

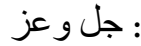

We.1685

: الوجود :

اوجود (copyist mistake - omission of letter alif) Schöm.

1. 10

: ومخالف :

(substantial variant) We. 1685, Schöm.

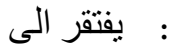

يفتقر الا (wrong grammar - perhaps caused by a dictation or hearing mistake) We. 1793

1.11

محل :

omitted Schöm.

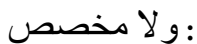

مخصص (substantial variant) We. 1793, Schöm.

: في ذاته ورلا

omitted We. 1793

: و ولا في صفاته

Dq. 97 و لا صفاته 
p. 3, 1. 1-5

: : الخمس ... الموجودات

(copyist mistake - dittography) We. 1793

1. 1

: ماله : افعاله

فعاله (substantial variant or copyist mistake - initial alif has been omitted)

We. 1685

: نفسبة :

(a substantial variant - which found its way into the text and contaminated the transmission) We. 1685, Pm. 105, Spr. 1956, Dq. 97, Schöm.,

Carullah Ef. 2125

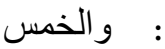

(grammar mistake?) We. 1685, 1793, Dq. 97, Schöm.

1. 2

: :

صفاة (copyist or hearing mistake - wrong orthography) We. 1685

1. 3

:

We. 1685

We. 1793

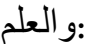

Schöm.

1. 3-4

: : الممكنات ... بجميع

Copyist mistake (omission because of homoioarcton) We. 1793

1. 4

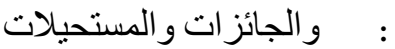

(substantial variant - inversion) We. 1793, Pm. 203, Schöm. 
1.5

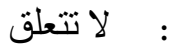

لا يتعلق (copyist mistake) Pm.105

لتقعلق (copyist mistake - omission) Spr. 1956

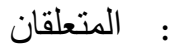

We. 1685

Dq. 97, Schöm.

1.6

: : : بحرف ولا صوت

(substantial variant - inversion of word order) Pm. 203

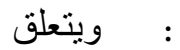

(substantial variant) Schöm.

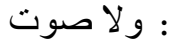

(copyist mistake involving letter alif) Dq. 97

1.7

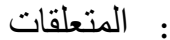

Dq. 97

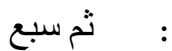

(substantial variant) Pm. 203

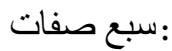

سبع (omission) We. 1685, 1793, Pm. 105, 203, Spr. 1956

Schöm.

1.8

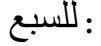

لسبع (copyist mistake - omission of letter lām) Dq. 97

: مقادر ا مريدا

قادر مريد فادر إن (grammatical mistake) Schöm. 
1.9

: مبصيرا :

(grammatical mistake) Schöm.

1. 10

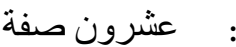

عشرون (omission) Dq. 97

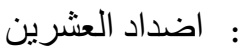

(grammatical mistake) We. 1685, Dq. 97

1. 11

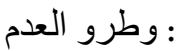

وطر او القدم (copyist mistake) We. 1685

(copyist mistake due to the nature of the Arabic script) Dq. 97

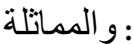

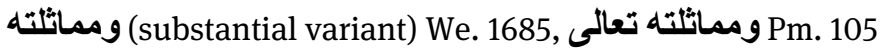

(!) و المماتلثنة) (copyist mistake) Schöm.

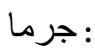

جزما (copyist mistake - diacritical mark mistake) Schöm.

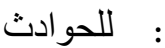

(copyist mistake) We. 179

1.12

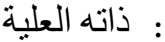

ذاته العقلية (substantial variant?) Dq. 97

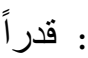

قدرً (copyist mistake - due to the nature of the Arabic script) Schöm.

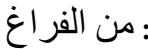

من الفراع (copyist mistake - diacritical mark omitted) Pm. 105

1.13

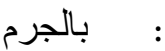

Pm. 203 
+ او محاذيا له او مرتشما في حياله (substantial variant - addition of words) Schöm.

للتجرم:

(copyist mistake) Schöm.

:

له جهة (substantial variant - omission of a single word) Pm. 105

1. 13-14

: بتقبد بمكان

يتقيد لمكان (copyist mistake) Pm. 203

1.14

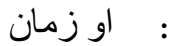

(substantial variant - inversion of word order) Pm. 203, Schöm.

: :تصفن :

يتصف (copyist mistake - diacritical mark variant) Schöm.

: :

تتصف (copyist mistake) Pm. 203, Dq. 97

1. 15

ذاته العلية بالصغر : (substantial variant - addition of words) Schöm.

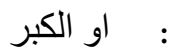

(substantial variant - addition of a preposition) We. 1793

: :

تتصف (copyist mistake involving a prefix and resulting in change of gender)

Pm. 203

: بالا غر اض اض

ذاته بالا غر اض : (copyist mistake) Schöm. 


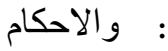

(substantial variant) Spr. 1956, Pm. 203

1. 16

:

(!) وكذاه) (spelling mistake, which perhaps occured during dictation) We. 1793

: بستحيل عليه

يستحيل (omission of a preposition) We. 1685

(substitution) Schöm.

ايضا + (addition) Carullah Ef. 2125

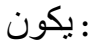

We. 1685

1. 17

: مفلة :

صفت (misspelling) Schöm.

1. 18

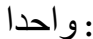

(substantial variant) Schöm.

: او يكون ... في ذاته

copyist mistake (omission caused by homoioarcton) Pm. 105

1. 19

: ماثله :

مماثل له (omission of a single word) Dq. 97

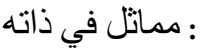

(copyist mistake - omission of a single word) We. 1793

:

(substantial variant) Schöm. 
p. 4, 1.1

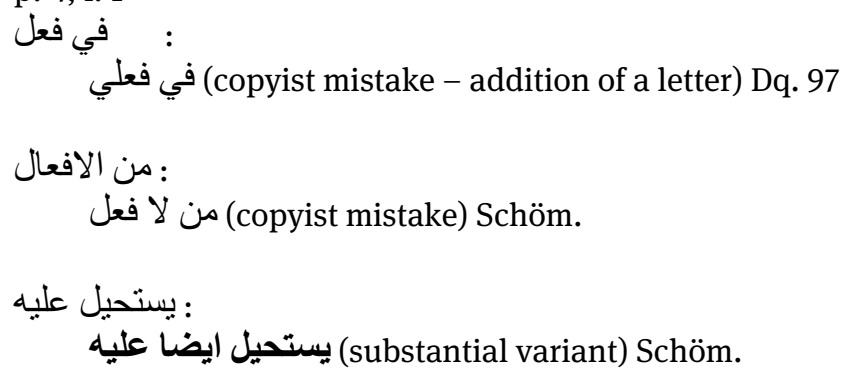

1. 2

: العجز عن :

(grammatically [lexically] impossible) We. 1685, 1793, Pm. 105, 203, Dq. 97, Carullah Ef. 2125

(copyist mistake - dittography) Schöm.

: وايجاد شي

(copyist mistake) Schöm.

: مع كر اهنه : مع اهنه

We. 1793

Schöm.

1.3

:

We. 1685

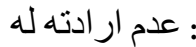

(omission) Spr. 1956

(misspelling) Schöm.

: الذهول

الدهول (diacritical mark mistake) We. 1685

1.4

: بالتعليل

بلتعليل (misspelling) We. 1685

Pm. 105 


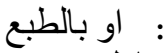

(substantial variant) We. 1685

(substantial variant) Pm. 105, 203, We. 1793

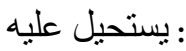

يستحيل ايضا عليه (substantial var.) We. 1685, Pm. 105, Spr. 1956, Dq. 97, Schöm., Carullah Ef. 2125

1.5

:

العمي:

(omission) Dq. 97

1. 5-6

: اضداد هذه الصفات :

(substantial variant) Spr. 1956, We. 1793, Pm. 203, Carullah Ef. 2125

اضداد الصفات (omission) We. 1685, Dq. 97, Schöm.

1.7

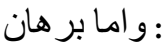

اما بر هان (omission) Pm. 105, Pm. 203, Spr. 1956

1.8

: لانه :

فلانه 105

(

له حدث (copyist mistake - different word/omission of a single letter) Spr. 1956

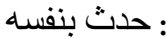

حدث لنفسه (substantial var.) We. 1685, 1793, Spr. 1956, Pm. 203, Schöm., Carullah Ef. 
1.9

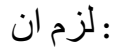

لزمان (copyist mistake - misspelling - or perhaps a hearing mistake) We. 1685

لزما ان (misspelling) Schöm.

:

رجحا Schöm.

1.10

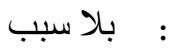

(copyist mistake) Schöm

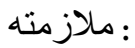

ملازمة We. 1685, Dq. 97, Schöm.

1.11

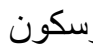

(substantial variant) Schöm.

1.12

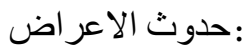

(copyist mistake - error of the eye) Pm. 203

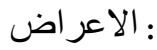

Schöm.

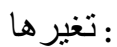

(misspelling) We. 1685

تغير (omission) Dq. 97

1.13

: وجوب القدم

We. 1685, Dq. 97

1.15

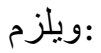

Schöm. 


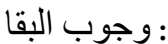

We. 1685

( )

(copyist mistake) Pm. 105, Spr. 1956

(orthographic mistake) Dq. 97

1.16

: يلحقه :

We. 1685

: لانتفى : لانفى :

لاتتفى (orthographic mistake) We. 1685

1.17

: حينئذ :

$\tau$ (abbreviation) Dq. 97

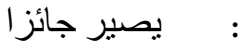

جائز (omission) Carullah Ef. 2125

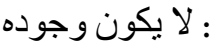

We.

:

يكون (omission of a word) Schöm.

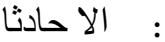

الحادثا

1.18

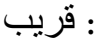

We. 1685

1. 18-p. 5, 1.2

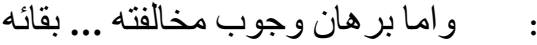

copyist mistake (omission caused by homoioarcton) Spr. 1956 


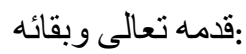

قدمها We. 1685, 1793, Dq. 97, Schöm.

قدمه تعالى Pm. 105, 203 (wa-baqā'ih has been added above the line but then crossed out again), Carullah Ef. 2125

1.19

: : : : : : مخالفته :

(orthographic mistake) Dq. 97

p. $5,1.1$

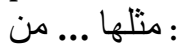

copyist mistake (saut du même au même) Schöm.

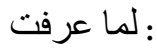

لما عرف We. 1685

قبل

omitted, Pm. 203

: وذلك ... و وجوب

omitted in We. 1793 and then added in the margin

1. 1-2

قدمه تعالى وبقائه:

قدمـ Dq. 97

1. 2

: مواما بر هان وجوب

We. 1793

1. 3

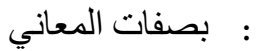

We. 1685, Dq. 97

:

لمعنوية Pm. 203, Dq. 97 
1.4

: و عز يجب

و عن يجب (copyist mistake) Schöm.

اتصنافه

(copyist mistake) Spr. 1956

بهما :

We. 1793

: ولو احتاج

Schöm.

1. 5

: بكان حادثا:

(addition, written above the line) Pm. 203

: كيف وقد :

We. 1685, 1793, Pm. 105, Dq. 97, Carullah Ef. 2125

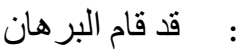

قد قدم البر هان

1. 7

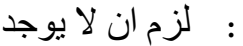

لماوجد (copyist mistake - saut du même au même) Schöm.

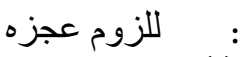

للزم(!) (misspelling) Pm. 203

1. 8

: :

Schöm.

ح

ح (abbreviation) Dq. 97

حينئذ ولو كان له تعالى مماثل في الو هية لزم ان لا يوجد شيء من الحو ادث mistake - homoioteleuton) Schöm. 


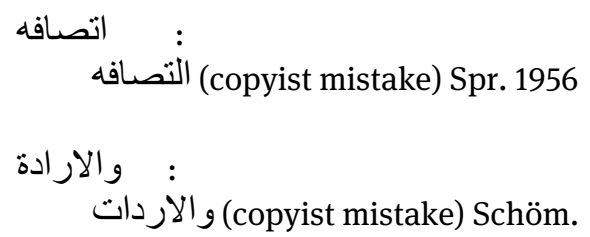

1. 10

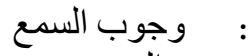

(copyist mistake - omission of a word) We. 1685

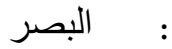

(copyist mistake - change of the consonantal skeleton) Dq. 97

\section{11}

فالكثاب

We. 1685, Spr. 1956

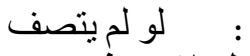

(substantial variant) Spr. 1956

1. 11-12

: : بها .... : بتصف

copyist mistake - omission caused by homoioteleuton) Dq. 97

\section{13}

: كون :

Dq. 97

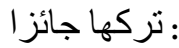

نركها جائز (copyist mistake - grammar mistake) We. 1685

1.14

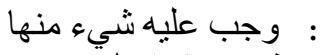

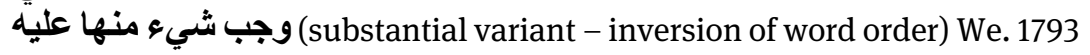

1.15

: مستحيلا :

Schöm. 
1. 17

وتبليغ

و (copyist mistake - grammar mistake) Schöm.

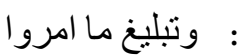

Omission caused by homoioteleuton, We. 1793

بتبليغه

Pm. 105, Spr. 1956, Dq. 97, Carullah Ef. 2125

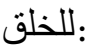

Schöm.

: و ويستحبل :

Schöm.

1. 18

: : :

صفاة We. 1685

:و وهي

وWe. 1685

p. $6,1.1$

: : : :

(misspelling or defective orthography) Schöm.

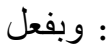

(copyist mistake - grammar) We. 1685, Spr. 1956

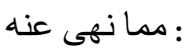

ما نهى عن : 1685

: نهي تحريم

omitted, Spr. 1956 
1.2

: :

و We. 1685, Spr. 1956, Pm. 203, Dq. 97, Schöm., Carullah Ef. 2125

Los:

من من Pm. 203

امروا :

امرواو (dittography of ending waw - alif) Dq. 97

بتبليغه

We. 1685

: للخلق :

omitted, Schöm.

1. 3

: الأي لا تؤدي

(orthographic mistake) We. 1685

1.4

: و ماما بر هان

اما بر هان Pm. 203, Carullah Ef. 2125

1.5

صدقهم

صدق الرسل Schöm.

: فلانهم :

فلانه Dq. 97

: لو لم لم يصدقوا

Schöm.

1.6

: لتصديقه :

Pm. 203 
ب : بالمعجزة

Carullah Ef. 2125

1. 7

: منز لة

omitted, Schöm.

1. 8

: وجوب الامانة

We. 1685

1. 8-9

لو خانو الو الو

لو كانو الو (copyist mistake - semantically impossible word variant) We. 1685

1. 9

: بفعل :

Schöm.

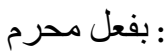

(omission) Pm. 105

: او المروره

(substitution of aw by wa-) We. 1685, Spr. 1956

1. 9-10

: في حقهم عليهم الصلاة و السلام

omitted, Schöm.

1. 10

:

امرنا Dq. 97, Schöm.

: بالاقتداء بهم

Schöm.

1. 11

: في اقو الهم

(misspelling) Schöm. 
: : : : :

Pm. 105, We. 1793 و افعالهم وسكوتهم

: بفعل محرم

We. 1685, 1793, Spr. 1956, Pm. 203, Dq. 97, Schöm., Carullah Ef. 2125

1. 12

: بر هان وجوب الثالث:

(omission) We. 1685

: :

Dq. 97

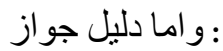

(inversion) Pm. 203

1.13

: : البشرية :

We. 1793

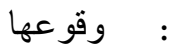

(copyist mistake - diacritical mark mistake) Schöm.

1.14

لتعظبم

Spr. 1956, Dq. 97, Schöm.

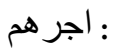

Spr. 1956 اجور هم : (copyist mist

اجرمه (copyist mistake) Schöm.

: للتسلي من :

We. 1685, 1793, Pm. 105, Spr. 1956, Carullah Ef. 2125

Spr. 1956

Schöm.

: :

عن الدنيا Schöm 
1. 14

و التنبيه

(substantial variant) Schöm., Spr. 1956

Pm.105, Dq. 97, Carullah Ef. 2125

1. 15

ل

We. 1685, 1793, Pm. 105, 203, Dq. 97, Carullah Ef. 2125

(Spr. 1956

Schöm.

رضاه بهاه :

رضاه الله بهاه Schöm.

جز :

جز 97

1. 15-16

: الانبيائه و اوليائه :

لاوليائه We. 1685, Pm. 105, 203, Spr. 1956, We. 1793, Dq. 97, Carullah Ef. 2125 الا لاوليائه (copyist mistake) Schöm.

1. 16

:

Dq. 97

1. 17

: :

We. 1685

: : :

Schöm

العقائد

(!) العناية) Dq. 97

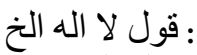

We. 1685 
1. 18

: الالله :

لا لا لهاله We. 1793

الا اله(!) Schöm.

\section{19}

و افتقار

و افتقر (copyist mistake) We. 1685

: : ماسواه

ما عداه Schöm., Carullah Ef. 2125

p. $7,1.1$

: لا مستغني

لا مستغنيا Pm. 203, Schöm.

ومفتقر

I ومفتقر Pm. 105, 203, Carullah Ef. 2125

او مفتقر

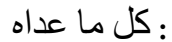

كل ما عاداه (copyist mistake - orthography) Spr. 1956

1. 2

: فهو بوجب :

Dq. 97

: :

(!)(copyist mistake - diacritical mark mistake) Schöm.

1. 3

: و القيام

Pm. 203

1. 3-4

: و القيام بالنفس

We. 1793, Schöm. 
1.4

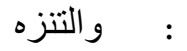

(perhaps a dictation/hearing mistake) Spr. 1956

Schöm.

: : : : النقائص :

Dq. 97, Schöm.

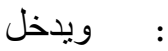

و ويََحَل

: السمع له :

(copyist mistake - omission of a word) Schöm.

1. 5

و الكالم

شميعا بصير متكلم (copyist mistake) Schöm.

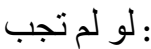

We. 1685

: : تجبب له :

(copyist mistake; orthography) Dq. 97

1. 6

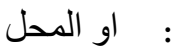

(substitution) We. 1685, Dq. 97

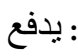

Dq. 97

1. 7

: :

هذه النقائص (addition) Pm. 203

: : : تنزيهه

تنز We. 1685, Pm. 105

Si Si Spr. 1956, We. 1793, Pm. 203, Dq. 97 (cacography), Carullah Ef. 2125 
: مي الافعال و الاحكام

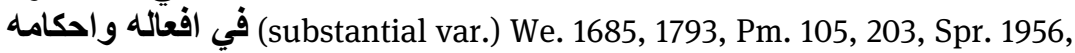
Dq. 97,

Carullah Ef. 2125

في افعالّه او احكامه (substantial var.) Schöm.

1.8

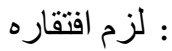

لز ام افتقاري (copyist mistake or hearing mistake) We. 1685

الى مان ما

Dq. 97

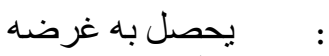

يحصل غرضه (copyist mistake - omission) We. 1685, 1793, Pm. 105, 203, Spr. 1956, Dq. 97, Schöm., Carullah Ef. 2125

1. 9

: الغني

(dittography) We. 1685

: بوخ خذ منه :

We. 1793

1. 11

: لو جو وجب

لو جب (copyist mistake - omission of a letter) Schöm.

: شيء منها

فعل شيء منها 105

عقل : عل :

عقلا + او استحال عقلا (addition) Spr. 1956

:

كاثوب (copyist mistake - orthography) We. 1685 
1. 12

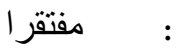

We. 1685

نفتقر (copyist mistake - orthography) Dq. 97

: ليتكمل به :

Spr. 1956

1.13

: : : :

كمال We. 1793

1. 15

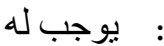

Spr. 1956

1. 16

: انتفى شيء منه منها :

انتفى شيء من هذه (substantial variant) Spr. 1956, We. 1793, Pm. 203, Dq. 97, Schöm., Carullah Ef. 2125

ان يوجد تعالى شيئًا :

1. 17

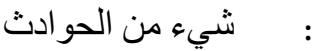

(substantial variant) Spr. 1956

1. 18

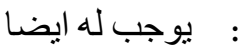

(inversion) We. 1685, 1793, Pm. 105, Dq. 97, Schöm.

-

We. 1793, Pm. 203

1. 19

:

We. 1685 
ثان (the first letter lacks the diacritical points [!]) Dq. 97

:

Dq. 97

(

Ge. 1685, Spr. 1956, Pm. 203, Schöm.

Dq. 97

حينئذ :

乙 (abbreviation) Dq. 97

p. $8,1.1-4$

: ويوخذ منه ايضا ... كل ماسو اه .

(copyist mistake - omission because of homoioteleuton) Spr. 1956

: ويوخذ منه منه

(addition) Carullah Ef. 2125

1.5

: تي اثر ما

في اثر هيا

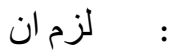

Dq. 97

: بيتغنى ذللك الاثر

يستغنى ذلك الثيء الاثر (copyist mistake - saut du même au même) Spr. 1956

1.6

: : عموما :

(!) عمو اه عما(copyist mistake) Schöm

1.7

: قدرت :

قدرة 1685 
1. 8

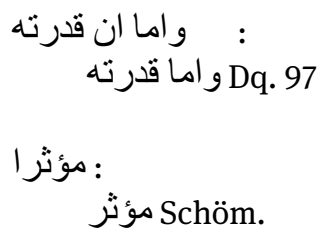


1. 11

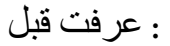

عرفت (copyist mistake - omission of a word) We. 1685, Spr. 1956, Dq. 97, Carullah Ef.

1.13

: الثلاثثة :

omission of a word, Dq. 97

1.14

: يجب :

تجب (copyist mistake) Pm. 203, Dq. 97

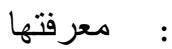

موفتها (copyist mistake - misspelling of individual letters) Dq.97

1.15

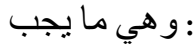

We. 1685

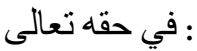

(substantial variant) Spr. 1956

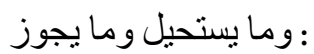

وما يجوز وما يستحيل :substantial variant - inversion) We. 1685 (end of Ms.),

Spr. 1956

1.16

الانبياء

(substantial variant - addition) Dq. 97

1. 17

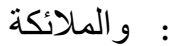

Omission (semantically possible), Pm. 203

1. 18

: جميع ذللك :

ذلك (substantial variant - omission of a word) Pm. 203 
p. $9,1.1$

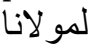

ولمو لانا (copyist mistake) Dq. 97

1.3

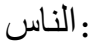

substantial variant - different word) Spr. 1956, Pm. 203

:

سكونهم Pm. 203

1.4

مخالفة

مخالفا (copyist mistake) We. 1793

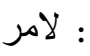

We. 1793

1.6

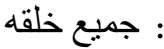

(substantial variant) Spr. 1956, Pm. 203, Carullah Ef. 2125

(copyist mistake) We. 1793

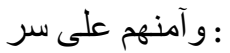

(substantial variant) Spr. 1956

1. 10

تضمن

نظمن (copyist mistake - orthography) Pm. 203

1. 11

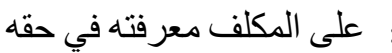

على المكلف من عقائد الايمان في حقه فئ (copyist mistake - omission of a word)

Spr. 1956, We.

1793, Carullah Ef. 2125

(substantial variant) Pm. 203 


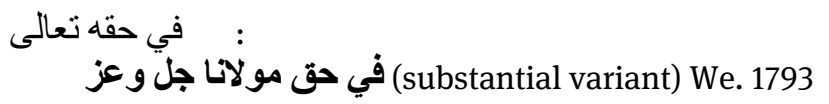

1.12

ولعلها

وقوله ولعلها (addition) Spr. 1956

1.13

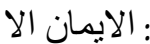

الا الايمان الا (copyist mistake) We. 1793

1.14

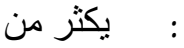

(copyist mistake - dittography) Spr. 1956

(؟كفر) (copyist mistake - change of the consonantal skeleton) We. 1793

1.15

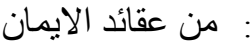

Omission (semantically possible), Pm. 203

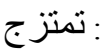

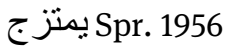

: بلحمه :

لحمه (copyist mistake) Spr. 1956

1.17

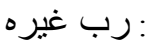

Carullah Ef. 2125 + و لا معبود سو اه

p. $10,1.1$

: وصلى الله

(copyist mistake - orthography) Spr. 1956

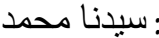

We. 1793 
1.2

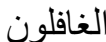

Spr. 1956 + وسلام على جميع الانبياء و المرسلين و الحمد لله رب العالمين

1.3

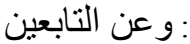

(substantial variant - addition) Spr. 1956, Carullah

Ef. 2125

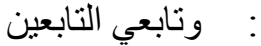

(copyist mistake - grammar) Spr. 1956

omission, We. 1793

تابعيهم Pm. 203

1. 3-4

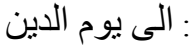

(copyist mistake - omission) Pm. 203

(subst. var.) We. 1793 
